\title{
Coating CoCrMo Alloy with Graphene Oxide and $\varepsilon$-Poly-L-Lysine Enhances Its Antibacterial and Antibiofilm Properties
}

\author{
Jianbin Guo ${ }^{1,2, *}$ \\ Guihua $\mathrm{Cao}^{3, *}$ \\ Xing Wang' \\ Wenhao Tang' \\ Weilong Diwu' \\ Ming Yan' \\ Min Yang' \\ Long $\mathrm{Bi}^{1}$ \\ Yisheng Han (D) \\ 'Department of Orthopedics, The First \\ Affiliated Hospital of Air Force Military \\ Medical University, Xi'an, People's \\ Republic of China; ${ }^{2}$ Department of Joint \\ Surgery, Hong-Hui Hospital, Xi'an \\ Jiaotong University College of Medicine, \\ Xi'an, People's Republic of China; \\ ${ }^{3}$ Department of Geriatrics, The First \\ Affiliated Hospital of Air Force Military \\ Medical University, Xi'an, People's \\ Republic of China \\ *These authors contributed equally to \\ this work
}

Introduction: With increases in implant infections, the search for antibacterial and biofilm coatings has become a new interest for orthopaedists and dentists. In recent years, graphene oxide (GO) has been extensively studied for its superior antibacterial properties. However, most of these studies have focused on solutions and there are few antibacterial studies on metal surfaces, especially the surfaces of cobalt-chromium-molybdenum (CoCrMo) alloys. $\varepsilon$-Poly -L-lysine ( $\varepsilon$-PLL), as a novel food preservative, has a spectrum of antimicrobial activity; however, its antimicrobial activity after coating an implant surface is not clear.

Methods: In this study, for the first time, a two-step electrodeposition method was used to coat GO and $\varepsilon$-PLL on the surface of a CoCrMo alloy. Its antibacterial and antibiofilm properties against $S$. aureus and E. coli were then studied.

Results: The results show that the formation of bacteria and biofilms on the coating surface was significantly inhibited, GO and $\varepsilon$-PLL composite coatings had the best antibacterial and antibiofilm effects, followed by $\varepsilon$-PLL and GO coatings. In terms of classification, the coatings are antiadhesive and contact-killing/inhibitory surfaces. In addition to oxidative stress, physical damage to GO and electrostatic osmosis of $\varepsilon$-PLL are the main antibacterial and antibiofilm mechanisms.

Discussion: This is the first study that GO and $\varepsilon$-PLL coatings were successfully prepared on the surface of CoCrMo alloy by electrodeposition. It provides a promising new approach to the problem of implant infection in orthopedics and stomatology.

Keywords: graphene oxide, $\varepsilon$-poly-L-lysine, antibacterial, antibiofilm, electroplating

\section{Introduction}

The rapid increase in infections caused by bacteria and their biofilms has become one of the greatest potential threats to human health. Antibiotics have been a traditional weapon in the treatment of infections for many years, but the emergence of multidrug resistance (MDR) makes the situation worse. MDR is not just a national problem but a very complex global phenomenon. ${ }^{1}$ Biofilms play an important role in infection caused by MDR bacteria: they are communities of surface attachment formed by microorganisms. ${ }^{2}$ It produces an extracellular matrix that protects microbes from drugs and host immunity leading to persistent infections. ${ }^{3}$ Therefore, an effective way to combat MDR bacteria and biofilms is to develop new and effective antibiotics and/or therapeutics. ${ }^{4,5}$ However, it is very difficult to find novel and effective antibiotics to replace and/or supplement existing antibiotics. ${ }^{6}$ In recent years, various materials such as polymers, ${ }^{7,8}$ hydrogels,,${ }^{9,10}$ antimicrobial peptides (AMPs), ${ }^{11,12}$ inorganic nanomaterials ${ }^{13,14}$ and other materials have been extensively studied.
Correspondence: Yisheng Han; Long Bi Email drhanys@fmmu.edu.cn; bilong@fmmu.edu.cn 
For orthopaedic and dental surgeons, implant infections are often catastrophic. They are the result of improper disinfection and/or improper handling of the material resulting in bacterial reproduction and the formation of biofilms on the material surface. Medical implant infections caused by bacterial biofilms are a major clinical problem. ${ }^{3}$ Reducing implant infections has been a major challenge for orthopaedic and dental surgeons.

In recent years, a growing number of researchers have begun coating artificial implants to prevent infection. Coating materials can generally be divided into two categories: those that combine antibiotics with polymers and those that rely on the intrinsic properties of the material to kill or repel bacteria on the contact surface. ${ }^{15}$ The antimicrobial properties of material surfaces can be divided into four categories: anti-adhesive surfaces, contact-killing /inhibitory surfaces, releasing-killing/inhibitory surfaces, and remote controllable bactericidal surfaces.

Cobalt-chromium-molybdenum (CoCrMo) alloys have become one of the main materials for artificial joints due to their excellent mechanical properties, abrasion resistance, corrosion resistance, and good biocompatibility. ${ }^{16,17}$ In recent years, an increasing number of studies have been performed on the surface modification of CoCrMo alloys. However, most of this research focuses on biocompatibility and mechanical properties and there is little research on its antibacterial and antibiofilm modification. ${ }^{18-20}$

With the rapid development of nanoscience and nanotechnology, various antibacterial nanomaterials, such as metal nanoparticles, ${ }^{21}$ carbon nanotubes, ${ }^{22}$ graphene and their derivatives ${ }^{23,24}$ have been widely studied. Graphene oxide (GO) is a type of graphene with abundant oxygen bonds at the edges and defect sites, such as carboxyl groups $(-\mathrm{COOH})$, carbonyl groups $(-\mathrm{C}=\mathrm{O})$, and hydroxyl groups $(\mathrm{OH})$ on the access side. $\mathrm{GO}$ was first used in medical research as a drug delivery agent in $2008 .^{25}$ However, since the antibacterial activity of GO was first discovered in $2010,{ }^{26}$ its antibacterial properties have attracted extensive attention in medical studies.

$\varepsilon$-Poly-L-lysine ( $\varepsilon$-PLL) is a kind of natural polymer synthesized by microorganisms and is characterized by a peptide bond between a lysine carboxyl group and $\varepsilon$ amino group. ${ }^{27}$ It is widely used in various foods, medicines, and electronic products. ${ }^{28} \varepsilon$-PLL has been found to have broad-spectrum antibacterial activity, ${ }^{29}$ the minimum inhibitory concentration for bacterial growth is less than $100 \mu \mathrm{g} / \mathrm{mL}$, and it is soluble in water, biodegradable, and stable under high temperature, acid, and alkaline conditions. ${ }^{27}$

In this study, for the first time, we used electrodeposition to coat the surface of CoCrMo alloy with GO and $\varepsilon$ PLL. We then characterized the coating and tested the mechanical properties and hydrophilic and hydrophobic properties of the coating surface. We further compared the antibacterial and antibiofilm effects of each coating on $S$. aureus and E. coli in the hope of providing a new direction for clinical treatment of surface infections of CoCrMo alloy prostheses.

\section{Materials and Methods \\ Preparation of Materials CoCrMo Alloys Substrate Preparation}

Medical-grade CoCrMo alloys (Northwest Institute for Nonferrous Metal Research, Xi'an, China $\Phi=12 \mathrm{~mm}$, $\delta=2 \mathrm{~mm}$ ) are polished to a mirror grade with mechanical polishing and diamond paste. Before each experiment, CoCrMo alloy sheets were cleaned by ultrasonication in a sequence of deionized water - anhydrous ethanol - acetone - deionized water for 20 minutes for each step, followed by $\mathrm{N}^{2}$ stream drying.

\section{CoCrMo Alloys Modified with GO}

GO solution with a concentration of $100 \mu \mathrm{g} / \mathrm{mL}$ was used. The alloy sheet was placed at the anode as the working electrode and the graphite electrode placed at the cathode. The speed of the magnetic agitator was 500 RPM. The voltage of the DC stabilized power supply was increased to $20 \mathrm{~V}$ at a rate of 1 $\mathrm{V}$ per second for $10 \mathrm{~min}^{30}$ After the reaction, the sample was gently rinsed with deionized water and dried in an $\mathrm{N}^{2}$ flow. The modified sample surface is named as $\mathrm{CoCrMo} / \mathrm{GO}$.

\section{CoCrMo Alloys Modified with $\varepsilon-\mathrm{PLL}$}

To optimize the $\varepsilon$-PLL coating on the CoCrMo surface an orthogonal experimental table with 3 factors and 4 levels was used to design the experiment. The 3 factors and 4 levels are: $\varepsilon$-PLL concentration $0.05 \mathrm{~W} / \mathrm{V} \%, 0.1 \mathrm{~W} / \mathrm{V} \%$, $0.15 \mathrm{~W} / \mathrm{V} \%$, and $0.2 \mathrm{~W} / \mathrm{V} \%$; voltage: $5 \mathrm{~V}, 10 \mathrm{~V}, 15 \mathrm{~V}$, and $20 \mathrm{~V}$; time: $5 \mathrm{~min}, 10 \mathrm{~min}, 15 \mathrm{~min}$, and $20 \mathrm{~min}$. According to the experimental test table, a total of 16 tests are needed (Supplementary Materials Table 1). The alloy sheet was placed on the cathode as the working electrode and the graphite electrode placed at the anode. The speed of the magnetic agitator was 500 RPM. The voltage of a DC- 
regulated supply was increased at a rate of $1 \mathrm{~V}$ per second to the corresponding operating voltage. After the reaction, the alloy sheets were gently washed with deionized water and dried in an $\mathrm{N}^{2}$ flow. The modified sample surface is denoted as $\mathrm{CoCrMo} / \varepsilon$-PLL.

\section{CoCrMo Modified with GO and $\varepsilon$-PLL}

To make a composite coating of GO and $\varepsilon$-PLL on the surface of the CoCrMo alloy, we first made a GO coating according to method. Once again, we used the orthogonal table of 3 factors and 4 levels for the $\varepsilon$-PLL coating (Supplementary Materials Table 1). CoCrMo/GO was used as the cathode, and a graphite electrode was used as the anode, immersed in $\varepsilon$-PLL aqueous solutions of different concentrations. Electrodeposition took place at room temperature. The magnetic stirring speed was 500 RPM. After electrodeposition was completed at different voltages and times, the alloy sheets were removed and dried in a $\mathrm{N}^{2}$ flow at room temperature. The modified sample surface is marked as $\mathrm{CoCrMo} / \mathrm{GO} / \varepsilon-\mathrm{PLL}$.

\section{Characterization of Materials}

The surface morphology and topography of the material were observed by scanning electron microscopy (FE-SEM, S-4800, Hitachi High Technologies, Tokyo, Japan) and atomic force microscopy (AFM, Agilent 5500 SPM, USA). After the coating, the samples of each group were mechanically polished again to remove the surface coating then ultrasonic shock cleaning was used. The composition changes of the CoCrMo alloy elements in each group then were detected by scanning electron microscopy energy dispersive spectrometry (SEM-EDS). There were 3 samples in each group, and 3 points in each sample were taken to calculate the mean value for each element. Fourier transform infrared (FTIR) spectroscopy (VERTEX70 FTIR-Spectrometer, Bruker Optics, Germany) with an attenuated total reflection accessory (ATR) was used to analyse the chemical composition of the modified surface. Hydrophilicity and hydrophobicity were measured with a contact angle meter (DSA 30, Kruss, Germany) by dropping $4 \mu \mathrm{L}$ of ultrapure water onto each sample. There were 6 samples in each group, and 6 different measurement areas were selected for each sample. The mechanical characteristics of the coating surface were measured using a nanoindentation instrument (Agilent Nano Indenter G200, USA). There were 3 samples in each group and 3 data points were measured for each sample.

\section{In vitro Antibacterial Experiments Bacterial Culture}

Gram-positive Staphylococcus aureus (S. aureus ATCC 25923) and gram-negative Escherichia coli (E. coli ATCC 25922) were used to evaluate its antibacterial and antibiofilm activity. The two bacteria were incubated overnight in brain-heart infusion (BHI, Solarbio, China) medium. Before each study, the two bacteria were adjusted to $10^{6} \mathrm{CFU} / \mathrm{mL}$ using a turbidity meter. All subsequent bacterial cultures were completed in a $0.05 \%$ dioxide incubator. All alloy samples were sterilized using Go60 before bacterial culture.

\section{Field Emission Scanning Electron Microscopy (FE-SEM) Observation}

Four groups of samples were put into a 24-well plate (triplicate of each group). Then, $1 \mathrm{~mL}$ of $10^{6} \mathrm{CFU} / \mathrm{mL}$ bacterial suspension was added to each well and cultured for 24 and 48 hours. After that, samples of each group were gently removed and rinsed with $\mathrm{PBS}(\mathrm{pH}$ 7.2) 3 times and then fixed in $2.5 \%$ glutaraldehyde at $4{ }^{\circ} \mathrm{C}$ overnight. The samples were dehydrated with gradient alcohol for 10 min with each gradient and soaked in hexamethyldisilane for $30 \mathrm{~min}$. After drying, samples were sprayed with gold and observed with FE-SEM.

\section{Antibacterial and Adherent Rate}

At the end of cultivation samples of each group were removed and rinsed with PBS gently 3 times to remove nonadherent bacteria. The sample was then immersed in normal saline and vibrated in ultrasound for 10 minutes to separate the bacteria that adhered to the surface of the material. Gradient dilution was then performed, $10 \mu \mathrm{L}$ of bacterial suspension was taken and evenly coated on blood plate medium, and CFUs were counted after 24 and 48 hours of culture. The antibacterial rate and adhesion were calculated using the following formula.

$$
\begin{aligned}
& \begin{array}{l}
\text { Antibacterial } \\
\text { rate }(\%)
\end{array}=\frac{\mathrm{CFU}(\text { Control })-\mathrm{CFU}(\text { Experimental })}{\mathrm{CFU}(\text { Control })} \\
& \text { Adhesion rate }(\%)=\frac{\mathrm{CFU}(\text { Experimental })}{\mathrm{CFU}(\text { Control })}
\end{aligned}
$$

\section{Bacterial Viability Assessment}

The viability of bacteria on the surface of different materials was evaluated using an Alamar blue assay kit (Alamar Blue, A7631, Solarbio, China). After S. aureus and E. coli were cultured on different surfaces for 24 and $48 \mathrm{~h}$, respectively, samples were gently washed with PBS to 
remove unadhered bacteria. After that, $500 \mu \mathrm{L}$ of $10 \%$ Alamar blue was added to each sample surface and then cultured at $37^{\circ} \mathrm{C}$ for 2 hours. ${ }^{31}$ After that, $100 \mu \mathrm{L}$ medium was transferred to a 96-well black plate, and fluorescence intensity (FI) was detected by a fluorescence spectrophotometer (Biotek, Synergy H1, USA) at an excitation wavelength of $540 \mathrm{~nm}$ and emission wavelength of $590 \mathrm{~nm}$.

\section{Fluorescence Microscopy}

Fluorescence images of $S$. aureus and E. coli with different coatings were detected at 24 and $48 \mathrm{~h}$ using a FilmTracer Live \& Dead Bacterial Backlight Kit (ZeYe Biotechnology Inc, China). For both bacterial species, S. aureus and E. coli, staining dissolution was prepared by mixing $100 \mu \mathrm{L}$ of DMAO and $200 \mu \mathrm{L}$ of ETHD-III in a microcentrifuge tube and then adding $800 \mu \mathrm{L}$ of $0.85 \% \mathrm{NaCl}$ solution to mix thoroughly. After incubating the two bacteria on different surfaces for 24 and 48 hours, a $40 \mu \mathrm{L}$ stain was dropped onto each substrate and then stained for $15 \mathrm{~min}$ in a dark room at room temperature. After dyeing, the substrate was gently rinsed with deionized water. The fluorescence of bacteria was observed using laser scanning confocal microscopy (LSCM, Fluoview FV1000, Olympus Corporation, Tokyo, Japan). Green excitation light (488 nm) was used to observe living bacteria (intact membrane). Red excitation light (543 $\mathrm{nm}$ ) was used to observe dead bacteria (damaged membrane).

\section{In vitro Antibiofilm Experiments Fluorescence Staining of Biofilms}

The biofilm was stained with concanavalin A fluorescein isocyanate-conjugated (ConA-FITC C7642; SigmaAldrich Inc, USA) to evaluate the effects of each coating on the biofilm at 24 and 48 hours. ${ }^{32}$ After the two bacteria were incubated on different surfaces they were rinsed 3 times gently with PBS. Then, $1 \mathrm{~mL}$ of $2.5 \%$ glutaraldehyde was added to each well, they were placed in a refrigerator at $4{ }^{\circ} \mathrm{C}$ for $1.5 \mathrm{~h}$, and again rinsed gently 3 times with PBS. After that, $40 \mu \mathrm{L}$ of ConA-FITC at a concentration of $50 \mu \mathrm{g} / \mathrm{mL}$ was dropped on the substrate surface and incubated at room temperature for $30 \mathrm{~min}$. The bacteria were then observed under LSCM (signals acquired $488 \mathrm{~nm}$; emission $552 \mathrm{~nm}$ ).

\section{Crystal Violet Assays}

The biofilm formation of $S$. aureus and E. coli was quantified using a crystal violet (CV) assay. After bacterial culture according to the above methods to the corresponding time point the unadhered bacteria were gently washed off the material surface with PBS. Then, the biofilm was fixed with $2 \%$ formalin for 15 minutes at room temperature. The samples were then placed into a new 24-well plate, $1 \mathrm{~mL} 0.1 \% \mathrm{CV}$ stain (Sigma-Aldrich, USA) was added to each well for $5 \mathrm{~min}$ which were then washed 3 times with PBS to remove excess stain. One $\mathrm{mL} 95 \%$ ethanol was then added to each well and incubated for $15 \mathrm{~min}$. After incubation, $200 \mu \mathrm{L}$ of ethanol was transferred to a 96-well plate and the absorbance at $570 \mathrm{~nm}$ was measured using a spectrophotometer (Biotek, Synergy H1, USA).

\section{Observation of Bacteriostasis Zone}

To determine whether the antibacterial effect was affected by the release of certain substances, a method of observing the inhibition zone was adopted. The Mueller-Hinton agar plate (MH plate) was evenly coated with $1.5 \times 10^{8} \mathrm{~S}$. aureus and E. coli, then the groups of materials were gently placed into the $\mathrm{MH}$ plate and cultured in a $0.05 \%$ dioxide incubator. The formation of inhibition zone was observed continuously in each group.

\section{Intracellular Reactive Oxide Species Assay}

To investigate ROS levels in bacterial cells an intracellular ROS detection kit (Beyotime, China) was used according to the manufacturer's instructions. As in the previous experiment, $S$. aureus and E. coli were cultured on different substrate surfaces for $24 \mathrm{~h}$ and $48 \mathrm{~h}$ and the bacteria that did not adhere to the surface were gently washed off. The samples were transferred to a new 24well plate. Then, $500 \mu \mathrm{L}$ DCFH-DA $(10 \mathrm{mM})$ was added to each well and cultured at room temperature for $30 \mathrm{~min}$.

Finally, $100 \mu \mathrm{L}$ medium was transferred to a 96-well black plate and the fluorescence intensity corresponding to DCF was detected at an extinction wavelength of $485 \mathrm{~nm}$ and an emission wavelength of $535 \mathrm{~nm}$ using an enzyme label instrument. ${ }^{31}$

\section{Statistical Analyses}

Intragroup and intergroup differences were estimated using statistical analysis of variance and Fisher's protected least significant difference (LSD) test. SPSS 20.0 software was used for statistical analysis and $\mathrm{P}<0.05$ was considered significant. 


\section{Results}

\section{Surface Characterization}

\section{Appearance}

The CoCrMo alloy group was the control group, marked as CoCrMo. Our experimental materials were divided into four groups: $\mathrm{CoCrMo}, \mathrm{CoCrMo} / \mathrm{GO}, \mathrm{CoCrMo} / \varepsilon-\mathrm{PLL}$, and $\mathrm{CoCrMo} / \mathrm{GO} / \varepsilon-\mathrm{PLL}$. After coating, the surfaces of different groups of materials exhibited different colors (Figure 1). The CoCrMo alloy sheet presented silver white (Figure 1A). The $\mathrm{CoCrMo} / \mathrm{GO}$ presented a golden

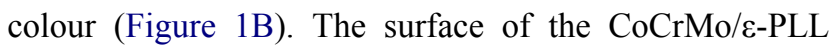
group is very similar to that of the CoCrMo alloy group which is also silvery white (Figure 1C). The $\mathrm{CoCrMo} / \mathrm{GO} /$ $\varepsilon$-PLL appears dark blue (Figure 1D).

\section{Two-Dimensional Morphology}

After completing 16 groups of tests using the parameters of the orthogonal experiment table, the surface morphology of each group of materials was observed using SEM (Figure 2). In $\mathrm{CoCrMo} / \varepsilon-\mathrm{PLL}$ (Figure 2A), all groups had a different appearance from the control group, as if there was a membrane overlay. When the voltage and the concentration were low the coating was in the thin film state, and with increasing concentration, time, and voltage, the coating was in the form of clusters. In the $\mathrm{CoCrMo} / \mathrm{GO} / \varepsilon$-PLL group (Figure 2B) it can be seen that the surfaces of some groups had almost no PLL coating (Ep 1). Some of the groups were orderly and granularly arranged and some were in a chaotic state (Ep 7,8,10). To ensure the uniformity of subsequent experiments, following our observation of these two groups of morphologies, we decided to use Experiment 2 ( $\varepsilon$-PLL $0.05 \mathrm{~W} / \mathrm{V} \%, 10 \mathrm{~V} 10 \mathrm{~min}$ ) to conduct subsequent studies, $\varepsilon$ PLL was uniformly coated on CoCrMo and GO surfaces and the GO surface not completely obscured.

After determining the parameters of each group, we observed the material surface of each group by SEM at different magnifications (Figure 3). The surface of the
CoCrMo alloy has obvious scratches (yellow arrow). On the $\mathrm{CoCrMo} / \mathrm{GO}$ group, we can see that GO is symmetrically attached to the surface of the alloy, and GO wrinkles on the surface of the alloy can be observed (blue arrow). After further modification of CoCrMo with $\varepsilon$-PLL, it can be seen that the surface of CoCrMo is a compact film (red arrow), different from that of bare CoCrMo alloy. However, on the CoCrMo/GO/e-PLL group we can see that GO wrinkles (blue arrow) and $\varepsilon$-PLL (green arrow) are uniformly distributed in granular form.

\section{Component Detection of CoCrMo Alloy Elements in Each Group}

To determine whether the composition of the CoCrMo alloy changes after electroplating, the samples of each group were polished and cleaned and the compositional changes of the CoCrMo alloy in each group were detected using SEM-EDS (Figure 4). We calculated the mean value of each element in each group and found that there was no statistically significant difference in each element between the groups (Table 1, $\mathrm{P}>0.05$ ).

\section{Three-Dimensional Topography}

To determine the three-dimensional structure of the material surface before and after modification, we used AFM to analyze the samples of each group (Figure 5). The surface of the CoCrMo alloy is heterogeneous, and scratches produced during the polishing process can be seen. However, after modification of GO and $\varepsilon$-PLL, a homogeneous organic layer can be seen covering the surface of the alloy. These scratches become blurred and even disappear, while wrinkles of GO and $\varepsilon$-PLL particles were observed. By further comparing the surface roughness of the four groups of materials (9 different $3 \mu \mathrm{m} \times 3 \mu \mathrm{m}$ regions were analysed for each sample), it was found that the surface roughness of the materials decreased significantly after modification with GO and $\varepsilon$-PLL. The roughness of the $\mathrm{CoCrMo} / \varepsilon-\mathrm{PLL}$ group is the lowest and that of the CoCrMo alloy group is

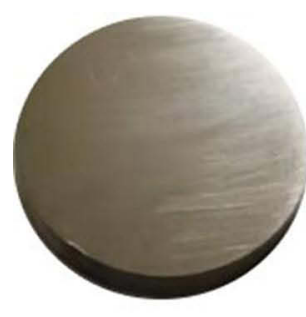

A

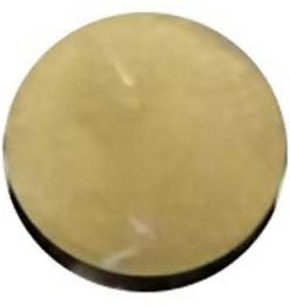

B

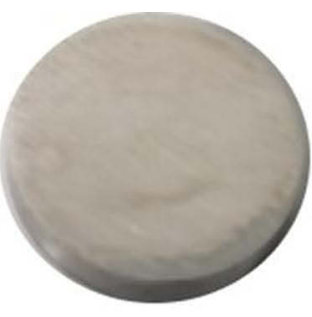

C

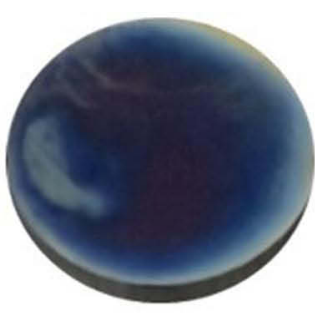

D

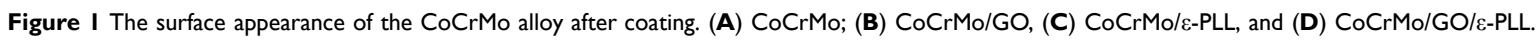




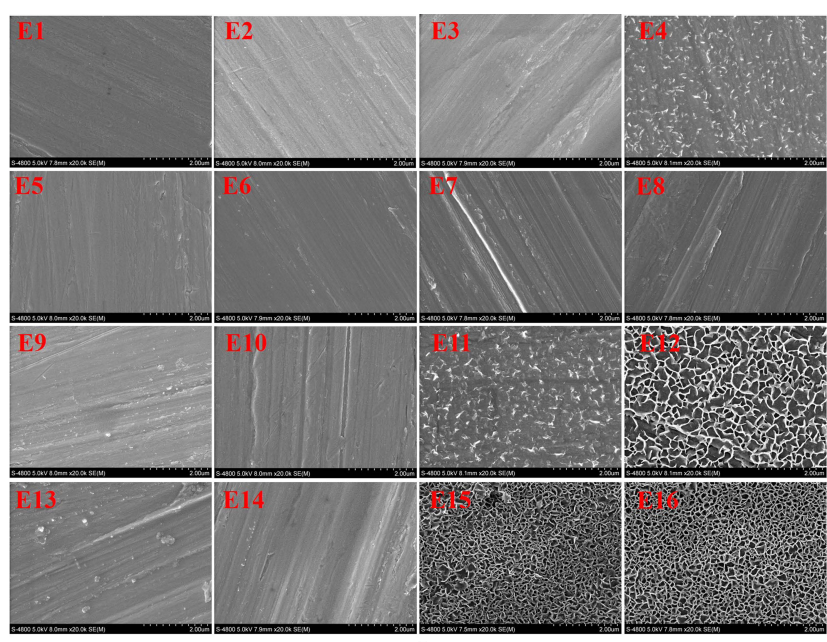

A

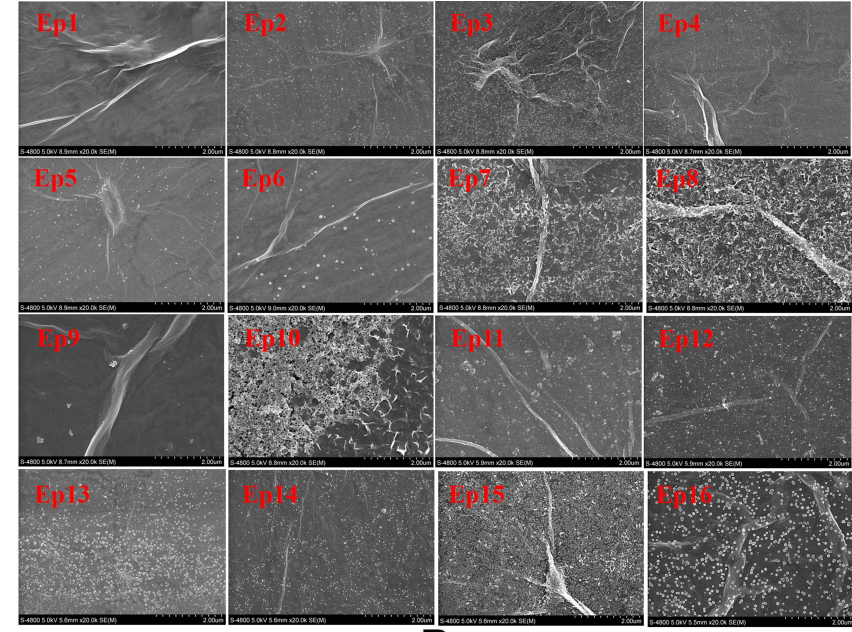

B

Figure 2 Orthogonal experimental results. (A) $\varepsilon$-PLL coating under different parameters (EI-EI6). (B) GO and $\varepsilon$-PLL composite coating under different parameters (Ep I-Ep I6).

the highest. There was no statistically significant difference between the $\mathrm{CoCrMo} / \mathrm{GO}$ group and $\mathrm{CoCrMo} / \mathrm{GO} / \varepsilon-\mathrm{PLL}$ group (Figure 6). GO and $\varepsilon$-PLL fill the gullies on the surface of the CoCrMo alloy and compensate for the unevenness of the CoCrMo alloy.

\section{Hydrophilic and Hydrophobic}

The wetting characteristics on the surfaces of the four groups are shown in Figure 7. Compared with the control group the contact angle of all the experimental groups was significantly reduced $(\mathrm{P}<0.05)$. CoCrMo/\&-PLL group had the best hydrophilicity. The change in wettability is consistent with the presence of the $\varepsilon$-PLL layer, as reported by other authors. ${ }^{33,34}$

\section{Mechanical Characteristics of the Coating Surface}

To verify the mechanical characteristics of the coating surface we used a nanoindentation instrument to measure each group. The elastic modulus and Vickers hardness of the control group were the highest, and the three experimental groups had different degrees of decline (Figure 8). We found that there were no statistically significant differences in elastic modulus except for the $\mathrm{CoCrMo} / \mathrm{GO}$ group. Vickers hardness results showed that compared with the CoCrMo group the difference between the $\mathrm{CoCrMo} / \mathrm{GO}$ and $\mathrm{CoCrMo} / \mathrm{GO} / \varepsilon$-PLL groups was statistically significant, while the difference in the $\mathrm{CoCrMo} / \varepsilon_{-}$ PLL group was not statistically significant (Table 2).

\section{FTIR-ATR Spectra Results}

After modification with GO and $\varepsilon$-PLL, each substrate was characterized by FTIR-ATR. The spectra in Figure 9 show the different types of oxygen functional groups in $\mathrm{CoCrMo} / \mathrm{GO}$. A broad $\mathrm{O}-\mathrm{H}$ stretching vibration peak appears at $3286 \mathrm{~cm}^{-1}$, strong $\mathrm{C}=\mathrm{O}$ peak at $1627 \mathrm{~cm}^{-1}$, a $\mathrm{C}-\mathrm{OH}$ stretching peak appears at $1265 \mathrm{~cm}^{-1}$, and C-O stretching peak at $1080 \mathrm{~cm}^{-1}$, confirming the presence of a large number of hydrophilic groups such as -OH, $\mathrm{COOH}$ and epoxides on the surface of the material after

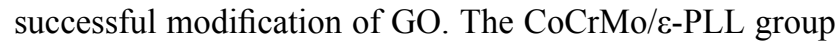
showed characteristic PLL absorption characteristics, $\mathrm{C}=\mathrm{O}$ stretching vibrations peak at $1645 \mathrm{~cm}^{-1}$ and characteristic $\mathrm{CH}_{2}$ stretching vibrations appeared at $2856 \mathrm{~cm}^{-1}$ and $2923 \mathrm{~cm}^{-1}$. The $\mathrm{CoCrMo} / \mathrm{GO} / \varepsilon$-PLL group showed both GO and PLL absorption characteristics, C-O stretching vibrations at $1140 \mathrm{~cm}^{-1}, \mathrm{C}=\mathrm{O}$ stretching vibrations at $1649 \mathrm{~cm}^{-1}, \mathrm{CH}_{2}$ stretching vibrations at $2856 \mathrm{~cm}^{-1}$ and $2922 \mathrm{~cm}^{-1}$ and $\mathrm{O}-\mathrm{H}$ stretching vibrations at $3288 \mathrm{~cm}^{-1}$. The results show that GO and $\varepsilon$-PLL were successfully coated on the surface of the CoCrMo alloy.

\section{Antibacterial Performances of the Modified Surfaces Morphology of Bacteria}

The morphology of bacteria on each surface was observed using FE-SEM. Figure 10 shows the morphological changes of the bacteria, illustrating the degree and properties of bacterial cell damage. On the surface of CoCrMo alloy, S. aureus can be seen growing well, with smooth, round, and complete cell morphology (A1, A2). The number of $S$. aureus on the GO surface was significantly reduced, some bacterial cell membranes were damaged, and cell perforation atrophy could be 


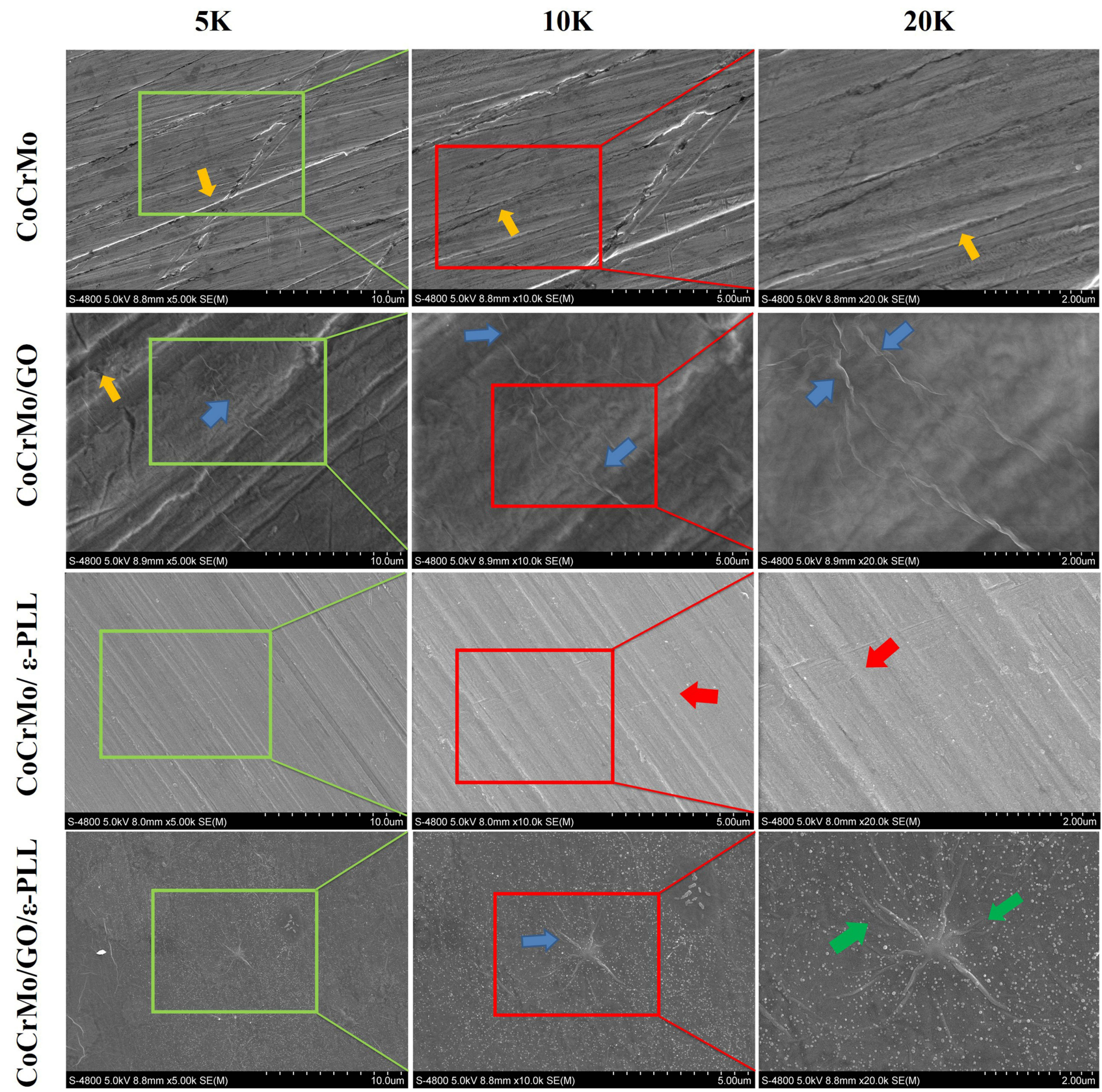

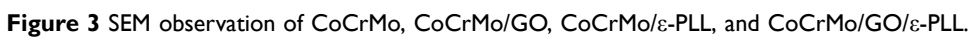

seen (B1, B2). On the CoCrMo/c-PLL surface, more S. aureus cell membranes burst and atrophied $(\mathrm{C} 1, \mathrm{C} 2)$. In $\mathrm{CoCrMo} /$ $\mathrm{GO} / \varepsilon-\mathrm{PLL}$ group, a significant increase in the number of bacterial cell deaths can be seen, and the cells are severely deformed, with breaking and bursting in addition to perforation and shrinking (D1, D2).

The situation was similar in E. coli. On the surface of CoCrMo, E. coli showed a short rod shape, intact cell membrane, and visible pili (E1, E2). On the GO surface, the number of $E$. coli was significantly reduced. The pili disappeared and part of the membrane rupture content was discharged (F1, F2). On the surface of $\varepsilon$-PLL the number of $E$. coli was also relatively reduced, the pili disappeared, with the cells ruptured and shrinking (G1, G2). On the surface of $\mathrm{CoCrMo} / \mathrm{GO} / \varepsilon$-PLL, it is almost impossible to see the normal shape of E. coli: cell fracture, atrophy, and cell membrane splitting content can be seen everywhere (H1, H2). 

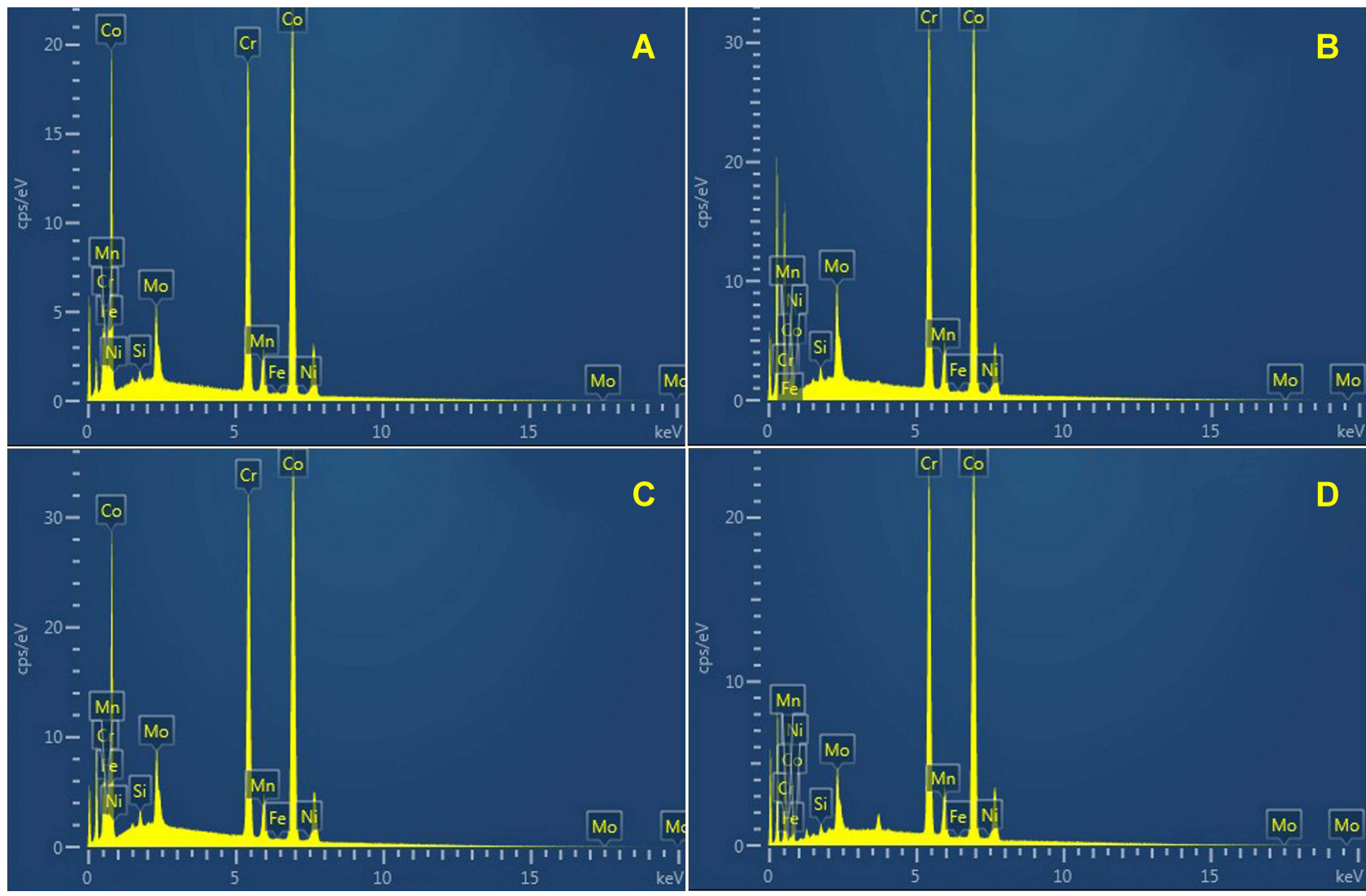

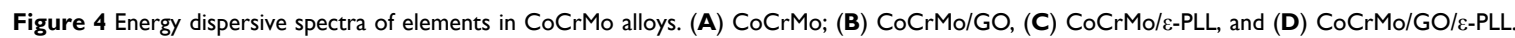

\section{Staining Results for Living and Dead Bacteria}

For cells without obvious morphological changes under SEM, to further determine whether they were dead, the living/dead bacteria staining method was used. In the CoCrMo alloy group, almost all exhibited green fluorescence, with a fluorescence intensity at 48 hours significantly higher than at 24 hours (Figure 11 A1, B1, C1, D1). Both green and red fluorescence existed in the $\mathrm{CoCrMo} /$ GO group, with the intensity of red fluorescence higher than that of green, and with an overall fluorescence intensity lower than that of the CoCrMo group (Figure 11 A2, $\mathrm{B} 2, \mathrm{C} 2, \mathrm{D} 2)$. Compared with the $\mathrm{CoCrMo} / \mathrm{GO}$ group the fluorescence intensity of the CoCrMo/PLL group was lower and the proportion of red fluorescence was higher (Figure 11 A3, B3, C3, D3). Almost all of the CoCrMo/ $\mathrm{GO} / \varepsilon$-PLL group showed red fluorescence, with the intensity of fluorescence significantly reduced, indicating that the little bacterial adhesion and with the adherent bacteria basically dead (Figure 11 A4, B4, C4, D4).

\section{Antibacterial and Adhesion Rate of Each Group}

The antibacterial activity and adhesion rate of $S$. aureus and $E$. coli were evaluated by plate counting method. After the bacteria on the surface of the material were fully

Table I Mean Values of Each Element in Each Group ( $P>0.05)$

\begin{tabular}{|c|c|c|c|c|}
\hline Elements & CoCrMo (wt. \%) & CoCrMo/GO (wt. \%) & CoCrMo/s-PLL (wt. \%) & CoCrMo/Go/e-PLL (wt. \%) \\
\hline $\mathrm{Si}$ & $0.45 \pm 0.05$ & $0.5 I \pm 0.074$ & $0.63 \pm 0.05$ & $0.39 \pm 0.01$ \\
\hline $\mathrm{Cr}$ & $28.19 \pm 0.24$ & $30.17 \pm 0.62$ & $28.52 \pm 0.07$ & $30.10 \pm 0.14$ \\
\hline$M n$ & $0.15 \pm 0.10$ & $0.22 \pm 0.06$ & $0.24 \pm 0.11$ & $0.25 \pm 0.13$ \\
\hline $\mathrm{Fe}$ & $0.16 \pm 0.10$ & $0.15 \pm 0.01$ & $0.13 \pm 0.04$ & $0.12 \pm 0.04$ \\
\hline Co & $64.40 \pm 0.37$ & $61.63 \pm 0.58$ & $63.95 \pm 0.10$ & $63.64 \pm 0.26$ \\
\hline $\mathrm{Ni}$ & $0.23 \pm 0.10$ & $0.17 \pm 0.12$ & $0.14 \pm 0.05$ & $0.14 \pm 0.01$ \\
\hline Mo & $6.44 \pm 0.07$ & $7.15 \pm 0.61$ & $6.42 \pm 0.08$ & $5.46 \pm 0.14$ \\
\hline
\end{tabular}



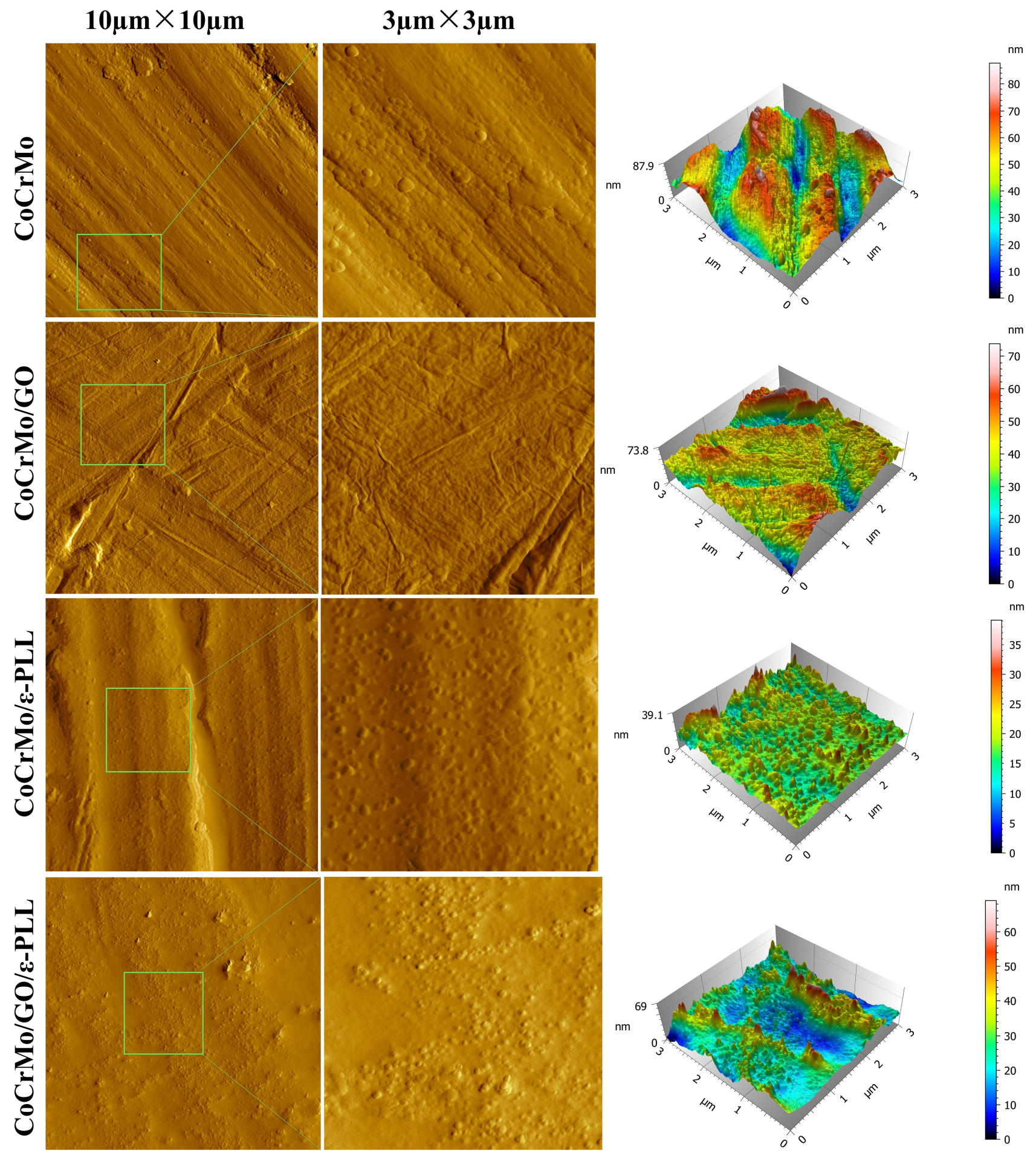

Figure 5 The surface morphology and three-dimensional structure of each group of materials.

shaken down by ultrasonic concussion, we diluted each group of bacteria $10^{4}$ times and then inoculated them on a blood plate medium. The results showed that there was a significant difference between the experimental group and the control group, with the $\mathrm{CoCrMo} / \mathrm{GO} / \varepsilon-\mathrm{PLL}$ group having the fewest colonies (Figure 12).
By further counting the number of bacterial colonies in each group, we found that the relative adhesion rate was the highest in the control group. When the relative adhesion rate of the control group was defined as $100 \%$, the first-day adhesion rates of $S$. aureus in the $\mathrm{CoCrMo} / \mathrm{GO}, \mathrm{CoCrMo} / \varepsilon$ $\mathrm{PLL}$, and $\mathrm{CoCrMo} / \mathrm{GO} / \varepsilon$-PLL groups were $22.8 \%, 15.35 \%$, 


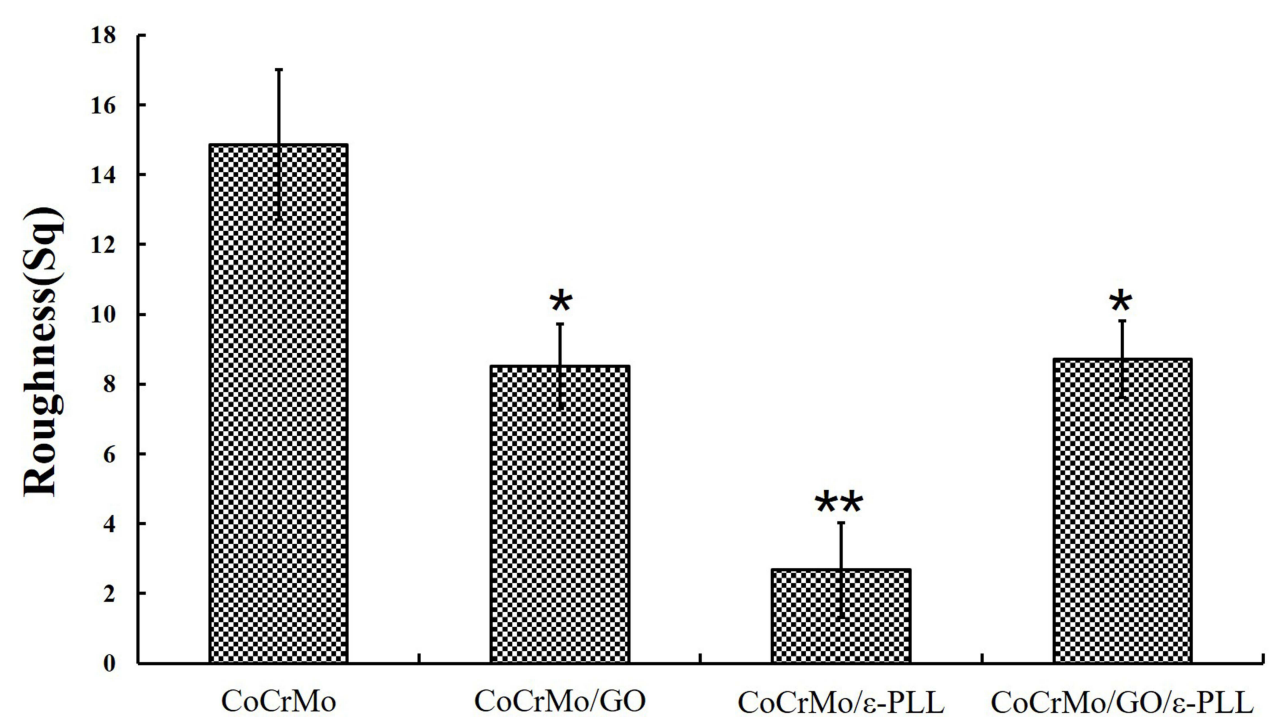

Figure 6 The comparison of four groups of roughness $(* \mathrm{P}<0.01, * * \mathrm{P}<0.00 \mathrm{I})$.

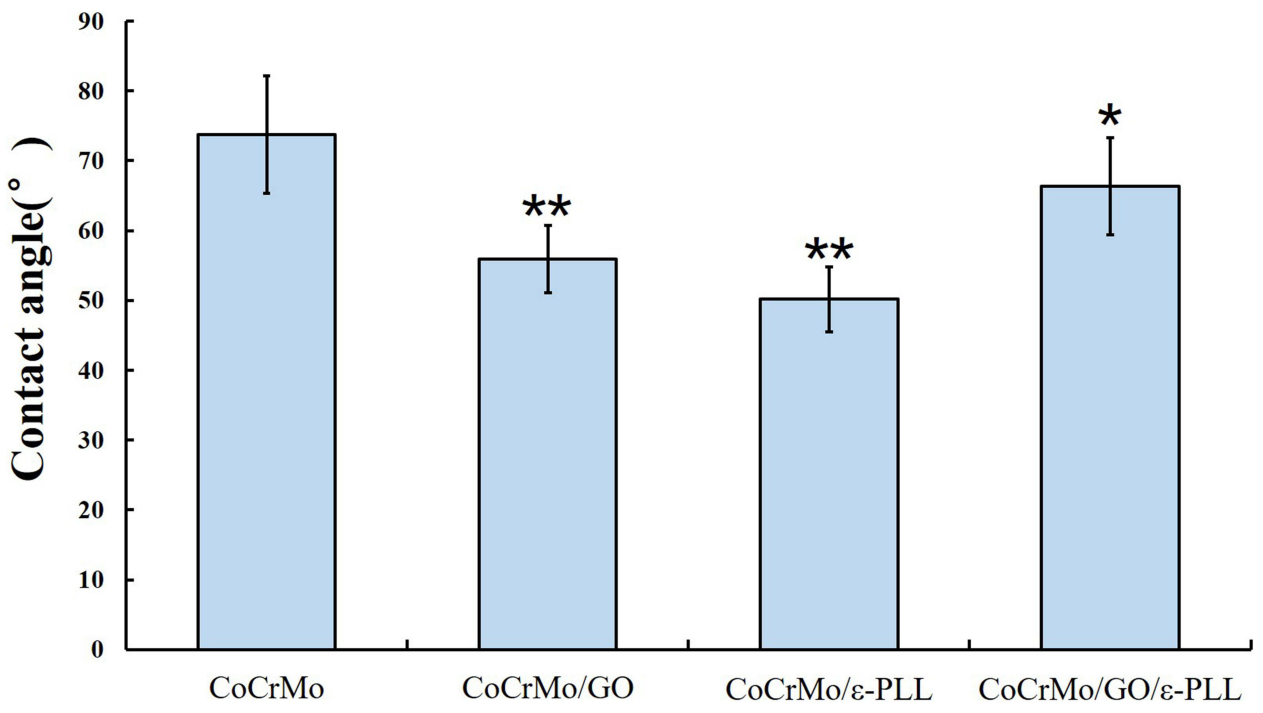

Figure 7 Comparison of the surface hydrophilicity of the four groups of materials $(* \mathrm{P}<0.05, * * \mathrm{P}<0.0 \mathrm{I})$.

and $8.86 \%$, respectively, and $26.6 \%, 10.8 \%$, and $6.37 \%$ on the second day (Figure 13A). These figures were $25.4 \%$, $20.3 \%$ and $10.6 \%$ for $E$. coli on the first day and $38.2 \%$, $23.4 \%$ and $12.5 \%$ for the second day (Figure 13B).

In our study of the antibacterial rate, we defined the antibacterial rate of the control group as 0 . We found that the antibacterial rate of the $\mathrm{CoCrMo} / \mathrm{GO} / \varepsilon$-PLL group was the best. On the first day, the antibacterial rates of the $\mathrm{CoCrMo} / \mathrm{GO}, \mathrm{CoCrMo} / \varepsilon-\mathrm{PLL}$, and $\mathrm{CoCrMo} / \mathrm{GO} / \varepsilon-\mathrm{PLL}$ groups against $S$. aureus were $77.2 \%, 84.65 \%$, and $91.94 \%$, respectively, and on the second day, they were $73.4 \%, 89.2 \%$, and $93.63 \%$, respectively (Figure 13C). The antimicrobial rates in $E$. coli on the first day were $74.6 \%, 79.7 \%$, and
$89.4 \%$, respectively, and those on the second day were $61.8 \%, 76.6 \%$, and $87.5 \%$ (Figure 13D).

\section{Bacterial Viability Assessment}

The viability of bacteria on the surface of each group was evaluated using Alamar blue staining. The results are presented in Figure 14. The $\mathrm{CoCrMo} / \mathrm{GO} / \varepsilon-\mathrm{PLL}$ group had the lowest fluorescence intensity, followed by the $\mathrm{CoCrMo} / \varepsilon-\mathrm{PLL}$ and $\mathrm{CoCrMo} / \mathrm{GO}$ groups. This means that bacterial activity in the control group was strongest, while the bacterial activity in each experimental group was weakened, and the weakest was $\mathrm{CoCrMo} / \mathrm{GO} / \varepsilon$-PLL. The pairwise comparison between each group was also statistically significant $(\mathrm{p}<0.05)$. 

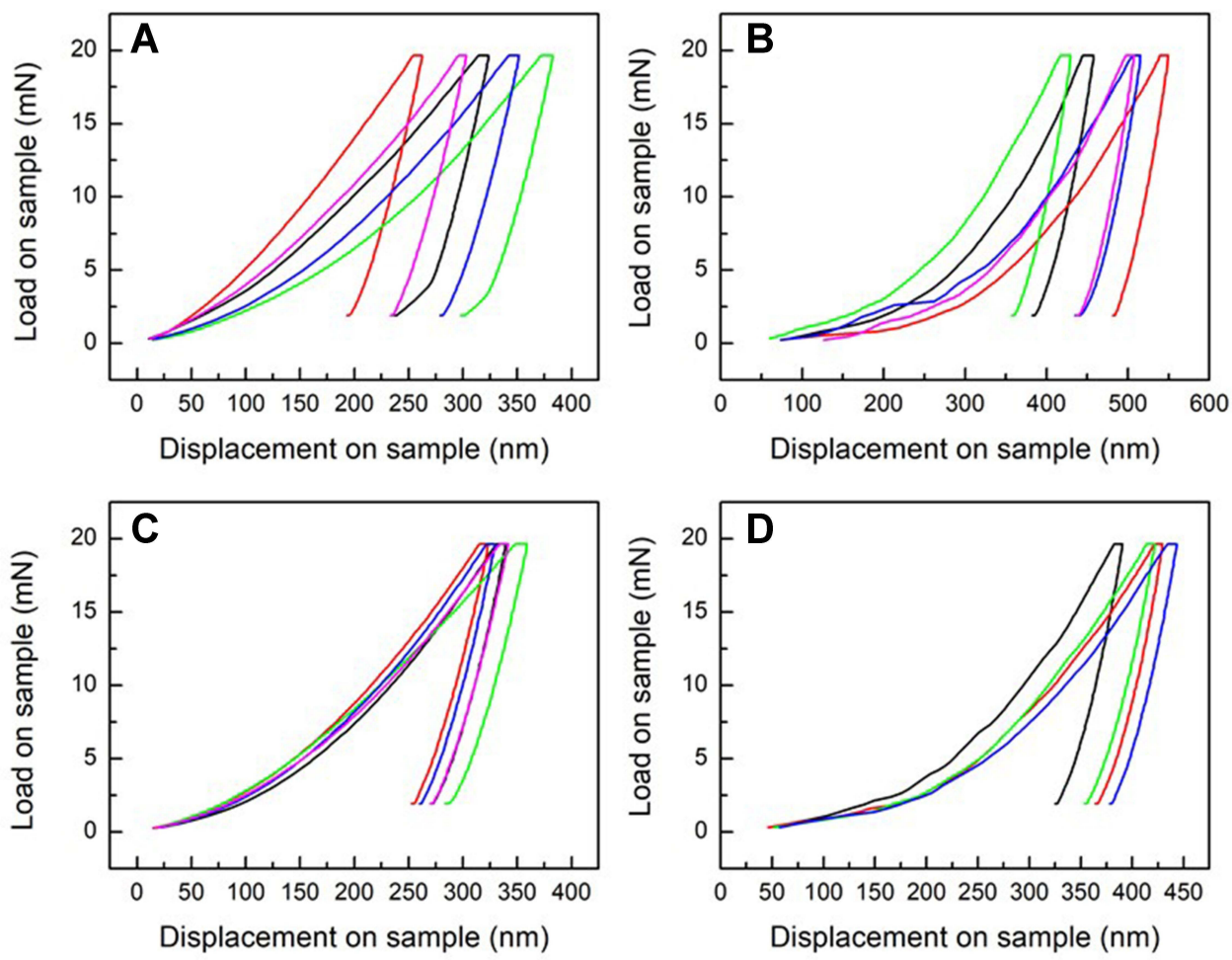

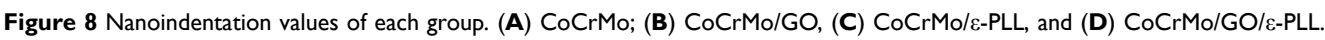

\section{Antibiofilm Performances of the Modified Surfaces}

The adhesion of bacteria to the surface of the material is initially reversible but becomes irreversible when the biofilm is formed. In this study, we observed the structures of biofilms under SEM (Figure 15). Our study shows that GO and $\varepsilon$-PLL have antibacterial effects on the surface of CoCrMo alloy. To further study whether they have antibiofilm effects, we carried out ConA-FITC staining and CV assays.

\section{Fluorescence Staining of Biofilms}

Glycocalyx in biofilms can be stained by ConA-FITC and fluoresce green in CLSM images. ${ }^{32}$ With this method, biofilms that are invisible even under electron microscopy can be observed. As seen in S. aureus (Figure 16A), a large amount of green fluorescence was observed on the surface of the control group and it was significantly stronger on the second day than on the first day. The green fluorescence on $\mathrm{CoCrMo} / \mathrm{GO}$ and $\mathrm{CoCrMo} / \varepsilon-\mathrm{PLL}$ was significantly reduced, and only a small amount of green fluorescence appeared on $\mathrm{CoCrMo} / \mathrm{GO} / \varepsilon-\mathrm{PLL}$ on the second day. The same trend was observed in $E$. coli (Figure 16B). In general, the fluorescence intensity of $E$. coli was weaker than that of $S$. aureus, indicating that $E$. coli is less capable of forming biofilms than $S$. aureus.

\section{Biofilm Quantification}

To further quantify the biofilms produced by each group, we performed a CV assay. The CV assay was first described by Christensen in 1985 and has been continuously improved to be applied to whole microorganism biofilm quantification. ${ }^{35}$ As described in the results (Figure 17), the absorbance of the control group was significantly higher than that of the other groups. Among the four experimental groups, the absorbance of the $\mathrm{CoCrMo} / \mathrm{GO} / \varepsilon-\mathrm{PLL}$ group was the lowest, while there was no significant difference between the $\mathrm{CoCrMo} / \mathrm{GO}$ and the $\mathrm{CoCrMo} / \varepsilon-\mathrm{PLL}$ groups. The overall absorbance of the $E$. coli group was slightly lower than that

Table 2 Values of the Elastic Modulus and Vickers Hardness $(* \mathrm{P}<0.05)$

\begin{tabular}{|c|c|c|c|c|}
\hline & CoCrMo & CoCrMo/GO & CoCrMo/e-PLL & CoCrMo/GO/\&-PLL \\
\hline Elastic Modulus & $268.652 \pm 56.436$ & $169.749 \pm 19.986 *$ & $249.261 \pm 14.875$ & $224.543 \pm 18.596$ \\
\hline Vickers hardness & $10.277 \pm 3.462$ & $3.892 \pm 0.836 *$ & $8.806 \pm 0.950$ & $5.254 \pm 0.627^{*}$ \\
\hline
\end{tabular}




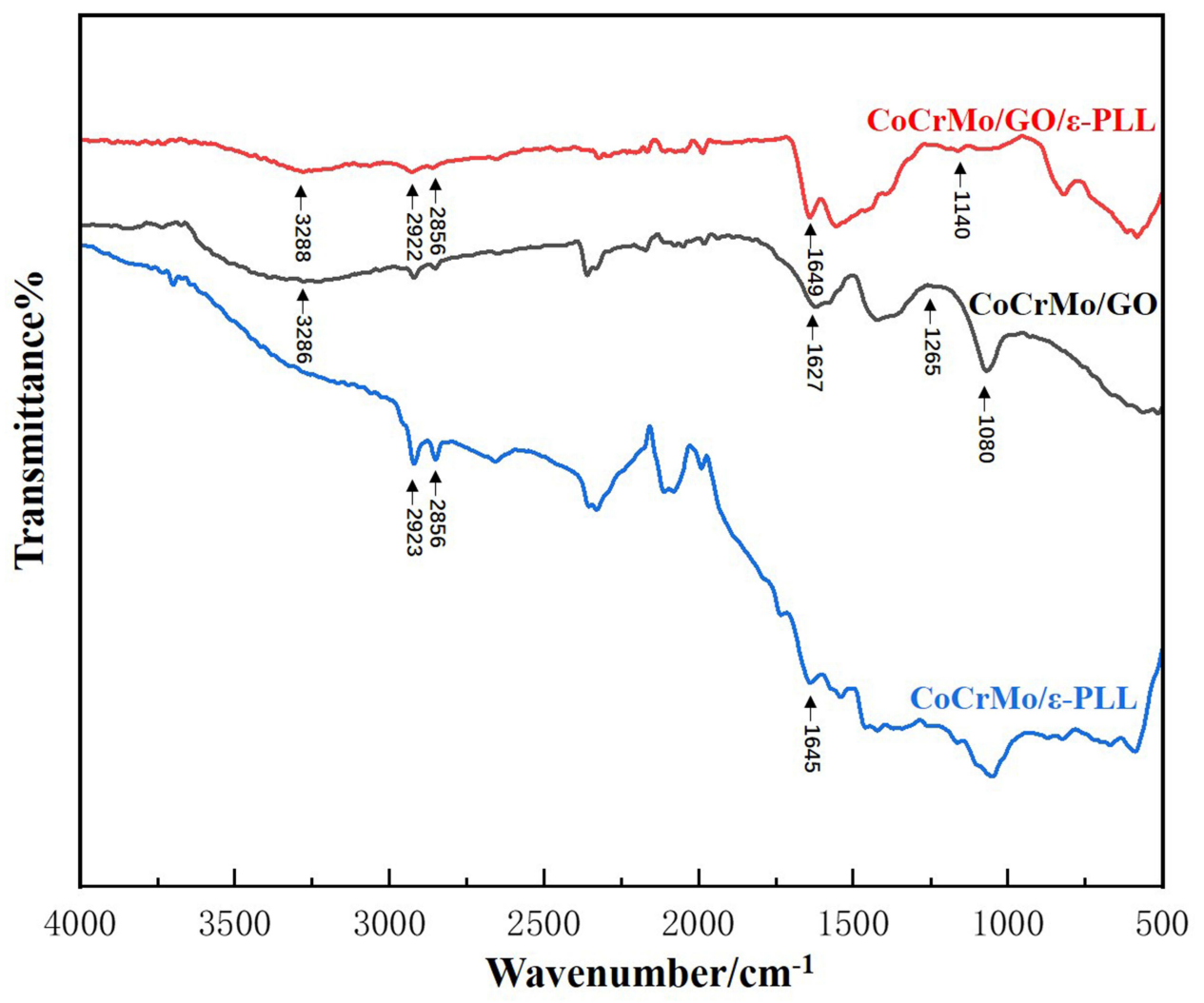

Figure 9 FTIR-ATR spectra of $\varepsilon$-PLL, GO, and GO/E-PLL on CoCrMo alloy.

of the $S$. aureus group, which also suggests that $E$. coli is less capable of producing biofilms.

\section{Determination of Inhibition Zone}

To determine whether the experimental group released something to cause its antibacterial activity, we used the method of observing inhibition zones. The continuous observation time was 3 days. The results showed that there was no inhibition zone in either group (Figure 18).

\section{Detection of ROS Fluorescence Intensity} To verify whether ROS affect the antibacterial activity of the material surface, we detected the ROS level in each group. The ROS level detection results of each group are shown in Figure 19. The results show that the ROS level of the $\mathrm{CoCrMo} / \mathrm{GO} / \varepsilon-\mathrm{PLL}$ group is significantly higher than that of the other three groups $(\mathrm{P}<0.001)$. However, there is no significant difference in ROS levels between the $\mathrm{CoCrMo} / \varepsilon$-PLL and the $\mathrm{CoCrMo} / \mathrm{GO}$ groups. The results indicate that ROS levels are one of the main antibacterial mechanisms of GO and $\varepsilon$-PLL.

\section{Discussion}

In the field of joint surgery and stomatology the advent of artificial joints and false teeth has greatly improved the quality of life of patients. As the population ages, the number of patients undergoing joint replacement and dental implants is increasing every year. ${ }^{36}$ Infections are a catastrophic complication in orthopedics and stomatology and are more difficult to manage due to the use of implant components. ${ }^{37}$ Mechanisms for bacterial adhesion, aggregation and biofilm formation on biomaterials include van der Waals, electrostatic, hydrophobic and glycoprotein-mediated forces, etc. ${ }^{37,38}$ Therefore, the development of materials that reduce the adhesion of pathogenic bacteria to the surface of the prosthesis and the formation of biofilms is fundamental to the reduction of periprosthetic infections. ${ }^{39,40}$ In recent years, a growing number of researchers have begun coating artificial implants to prevent infection.

Through a literature review, we know that both GO and $\varepsilon$-PLL are substances that have been demonstrated to have broad-spectrum antibacterial properties. However, most of the antibacterial studies focus on the solution, and few on 


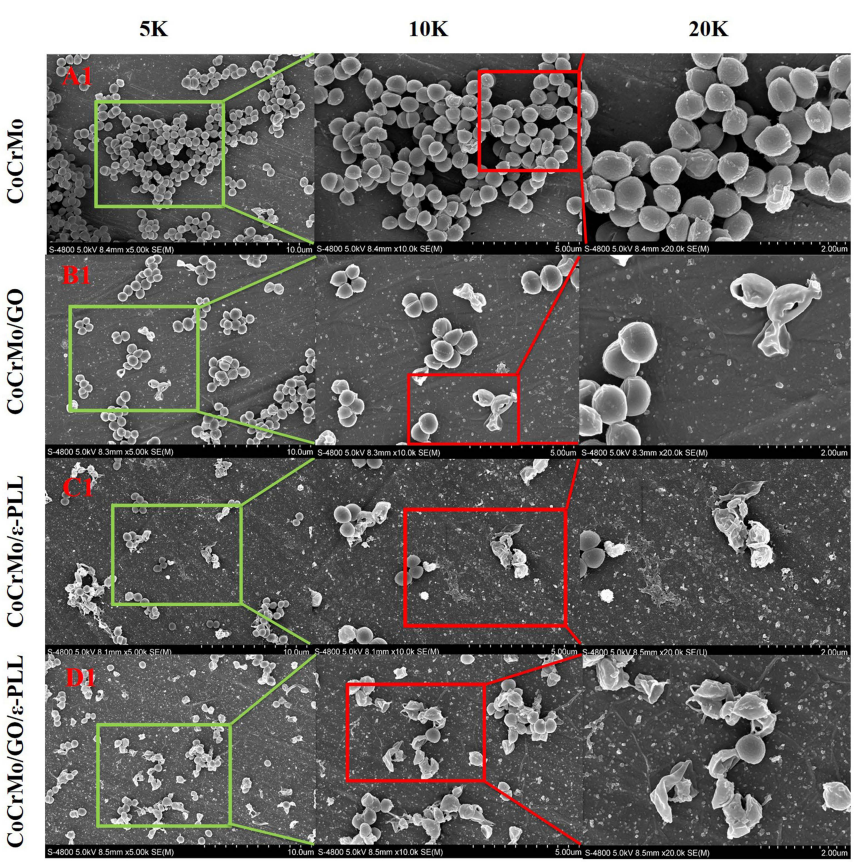

A

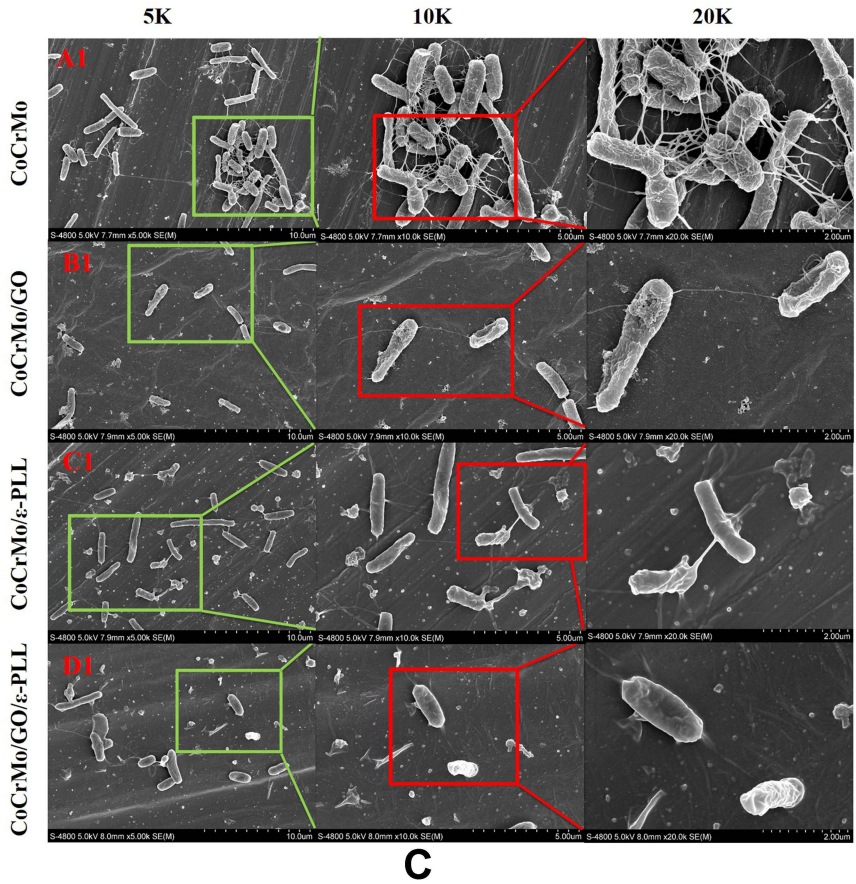

$5 \mathrm{~K}$

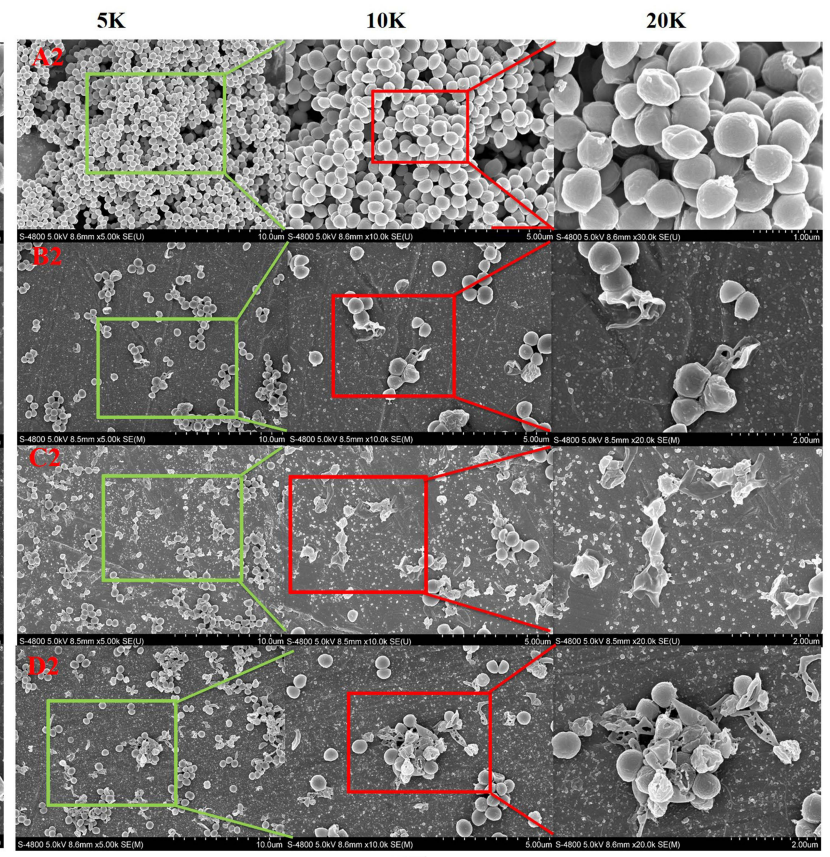

B

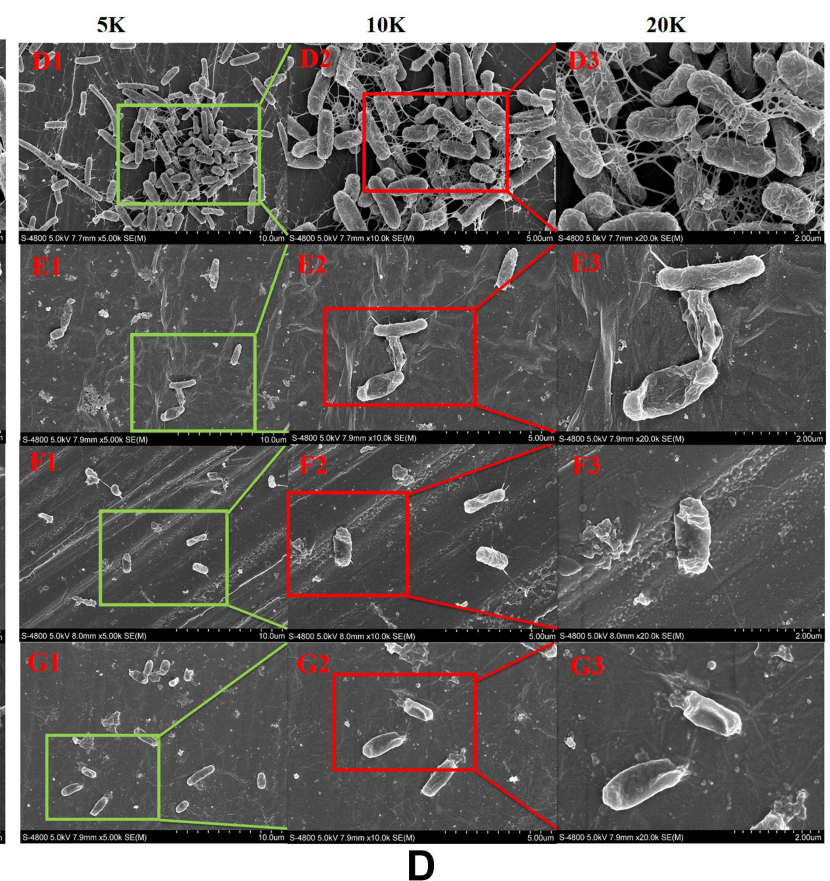

Figure 10 Morphology of S. aureus ((A) 24 h, (B) 48 h) and E. coli ((C) 24 h, (D) 48 h) on the surface of each group.

the surface of the alloy. GO in aqueous solution is negatively charged ${ }^{30}$ while $\varepsilon$-PLL is positively charged, ${ }^{41}$ making it possible to deposit GO and $\varepsilon$-PLL on alloy surfaces by electroplating. In this study, GO and $\varepsilon$-PLL were deposited on the surface of a CoCrMo alloy by electrodeposition for the first time. As the base material of the coating, the stability of CoCrMo alloy under voltage was one of the decisive factors in this study. Numerous literatures evidenced that CoCrMo alloys have high biological inertia and have been extensively used for artificial joints for many years. Likewise, it has been reported that CoCrMo alloys have inherently higher biological inertia than TiAlV alloys. ${ }^{42}$ One study involved nitriding the surface of CoCrMo alloys to increase their corrosion resistance. The 


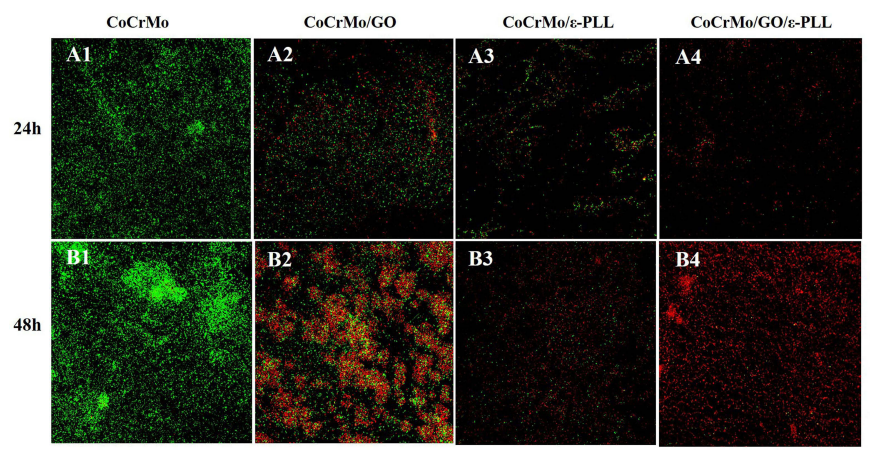

A

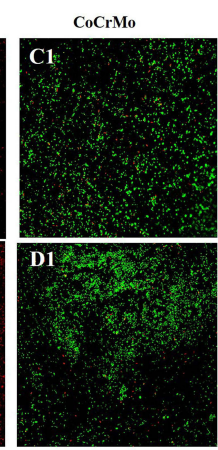

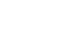

Figure I I Fluorescence staining of living and dead bacteria from different coating surfaces. (A) S. aureus. (B) E. coli.

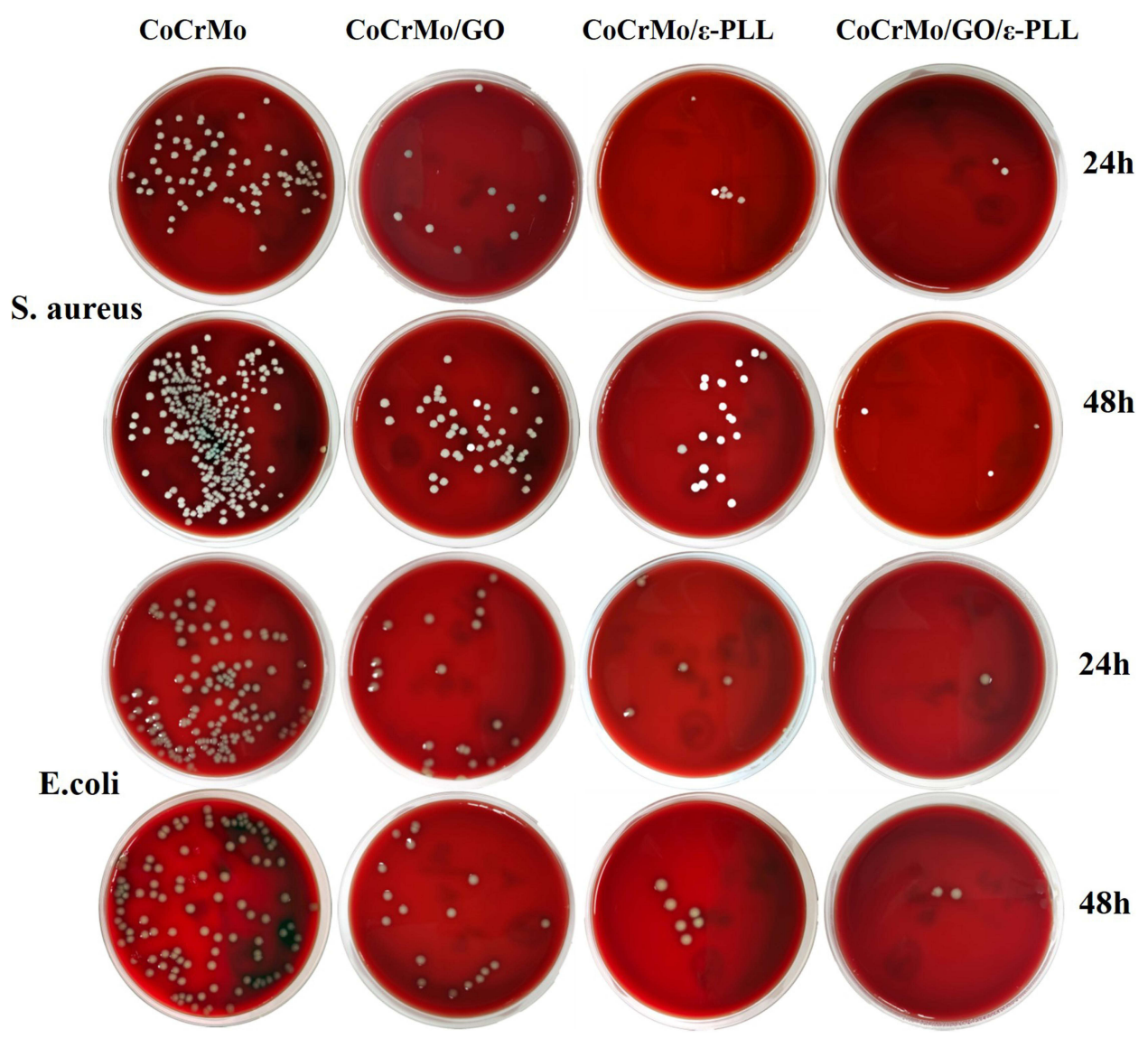

Figure 12 Bacterial colonies of S. aureus and E. coli of each group on the blood plate.

detailed method is to place the CoCrMo alloy at $400^{\circ} \mathrm{C}$ and load the voltage of $500 \mathrm{~V}$ to $1100 \mathrm{~V}$. After 4 hours, except for the addition of nitriding layer on the surface, no change in the components of the CoCrMo alloy was observed. ${ }^{19}$ In joint replacement surgery, surgeons apply a high-voltage, high-frequency current close to a metal implant to stop bleeding in adjacent blood vessels, and the voltages reach as high as $5 \mathrm{kV} .^{43}$ Also, the study in the effect of CoCrMo alloys under a high current electrotome showed that only minor damage was raised. Fortunately, in these reports no alteration in alloy components was found following high voltage treatment. In our present study, the voltage of $20 \mathrm{~V}$ only was used to conduct electrodeposition. Therefore, it is not possible to change the ion dissolution status of this alloy under such lower voltage. To further substantiate our claim, we carried out ultrasonic vibration 

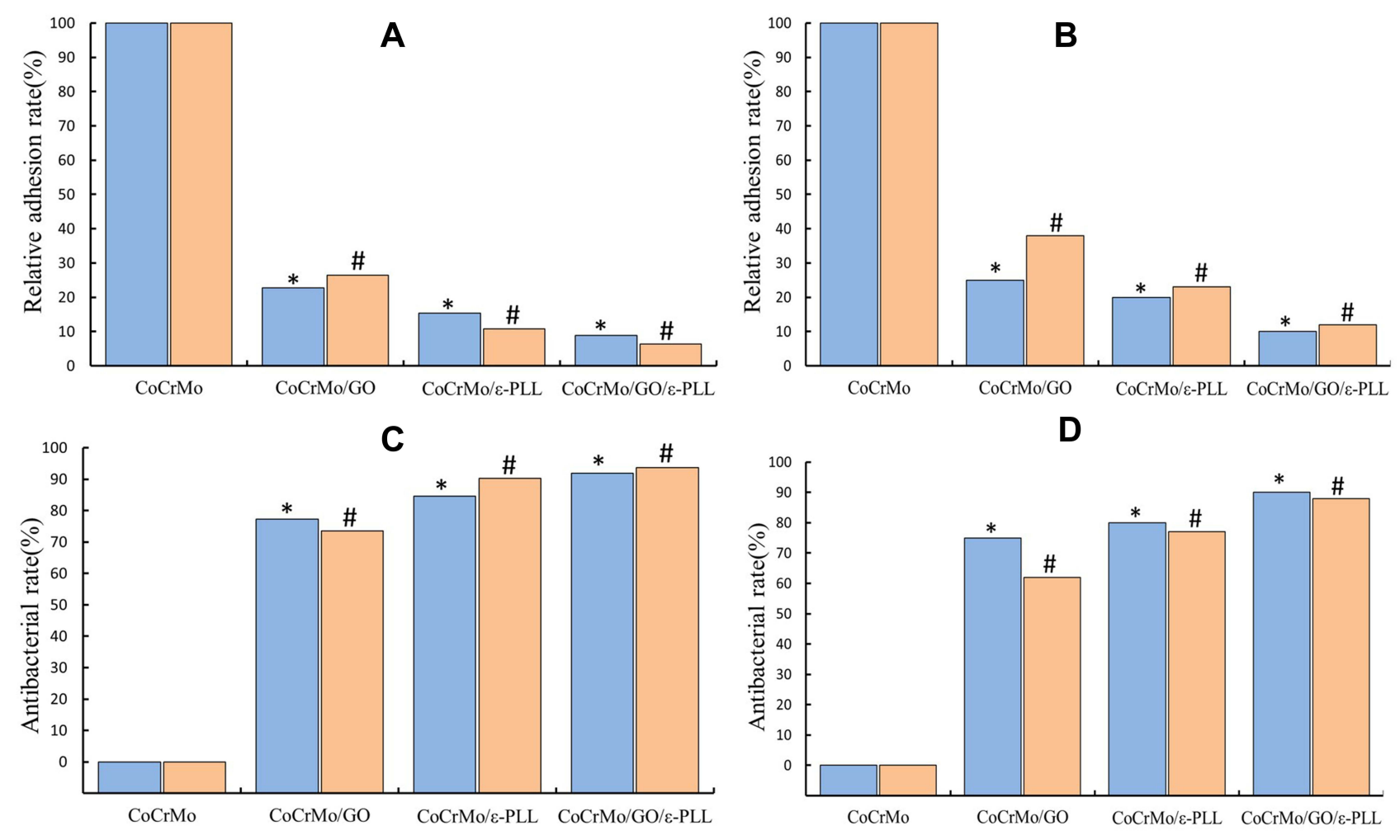

$\square$ The First day $\square$ The second day

Figure 13 The relative adhesion rate and antibacterial rate of each group. The relative adhesion rate of $S$. aureus $(\mathbf{A})$ and $E$. coli $(\mathbf{B})$ and the antibacterial rate of $S$. aureus $(\mathbf{C})$ and $E$. coli (D). *and \# $\mathrm{P}<0.001$.

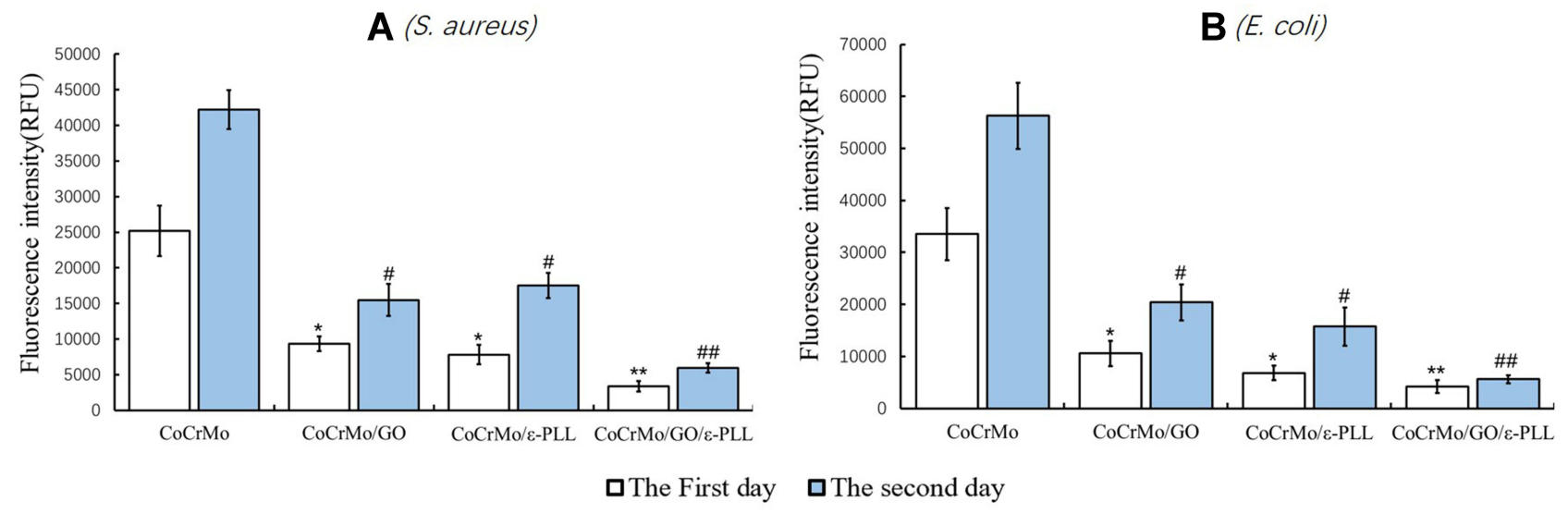

Figure 14 Fluorescence intensity of different samples (*and \# $\mathrm{P}<0.0 \mathrm{I}$, ** and \#\# $\mathrm{P}<0.00 \mathrm{I}$ ). (A) S. aureus. (B) E. coli.

cleaning and mechanical polishing again on the materials after the voltage action, and found no change in alloy components by means of SEM-EDS scanning.

After successful coating, the antibacterial and antibiofilm activities of GO and $\varepsilon$-PLL on the surface of CoCrMo alloy in vitro were systematically studied. We used SEM observation of cell morphology, living/dead bacteria staining, colony counting, Alamar blue staining, and other methods to study the antibacterial properties of each group of materials. The results show that GO and $\varepsilon$ PLL exhibit significant inhibitory effects on $S$. aureus and E. coli on the surface of CoCrMo alloy, leading to a decrease in bacterial cell aggregation and activity. In the $\mathrm{CoCrMo} / \mathrm{GO} / \varepsilon-\mathrm{PLL}$ group, the perforation, shrinking, 

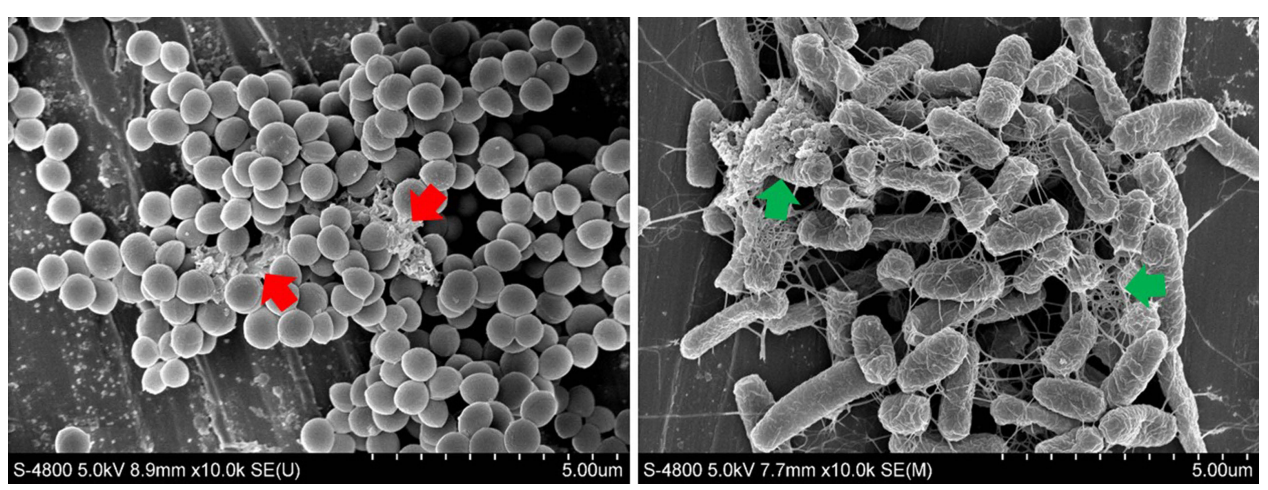

Figure 15 Biofilm structure of S. aureus (red arrow) and E. coli (green arrow).

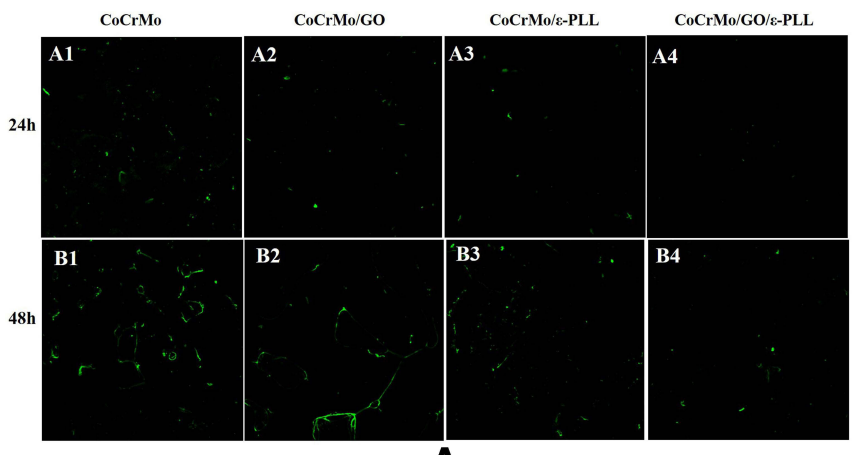

A

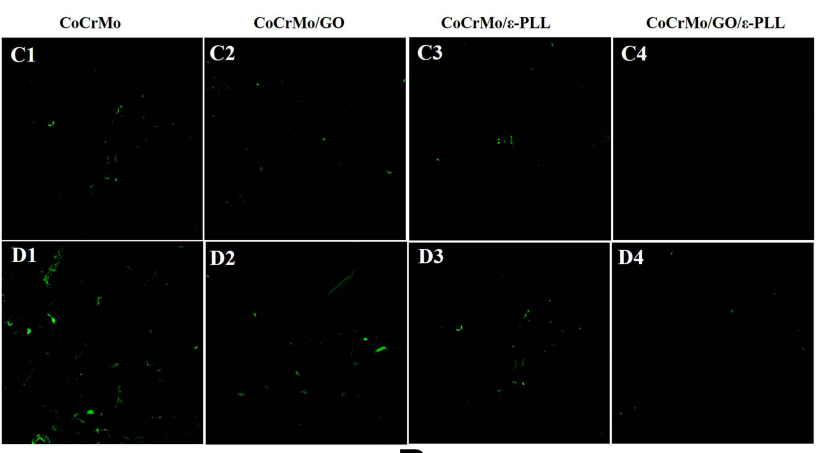

B

Figure 16 Results of biofilm staining. (A) S. aureus. (B) E. coli.
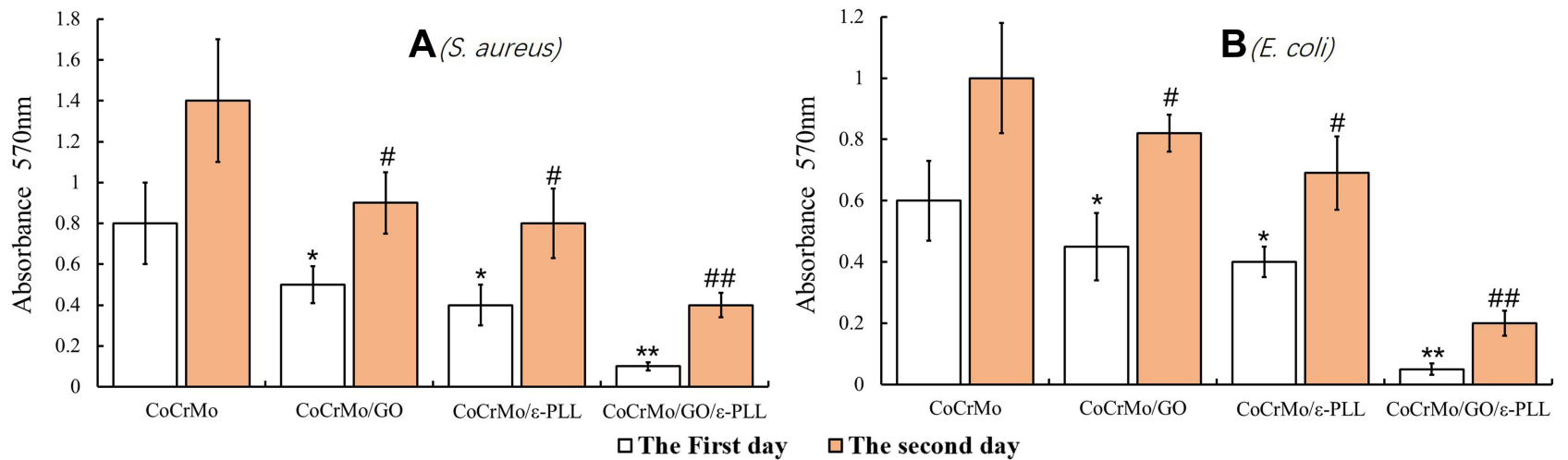

Figure 17 Results of $C V$ assay (*and \# P<0.05, ** and \#\# P<0.0I). (A) S. aureus. (B) E. coli.

atrophy, bursting, and cell membrane splitting of bacterial cells could be seen everywhere.

Biofilm formation is one of the most important events in the development of biomaterials infection and is also a difficult clinical problem. ${ }^{3}$ In general, it goes through several stages, including bacterial community development, maturation, and decomposition. ${ }^{37}$ Biofilm formation is fatal to the implant and is difficult to reverse because traditional treatments are often ineffective in treating biofilms. ${ }^{44,45}$ So, it is very important to prevent the formation of biofilm. To determine whether the experimental materials have an antibiofilm effect, we first observed the biofilms of $S$. aureus and E. coli using SEM and then further observed the differences in biofilms among the groups using ConA-FITC staining. After the differences were observed, the $\mathrm{CV}$ staining method was used to 

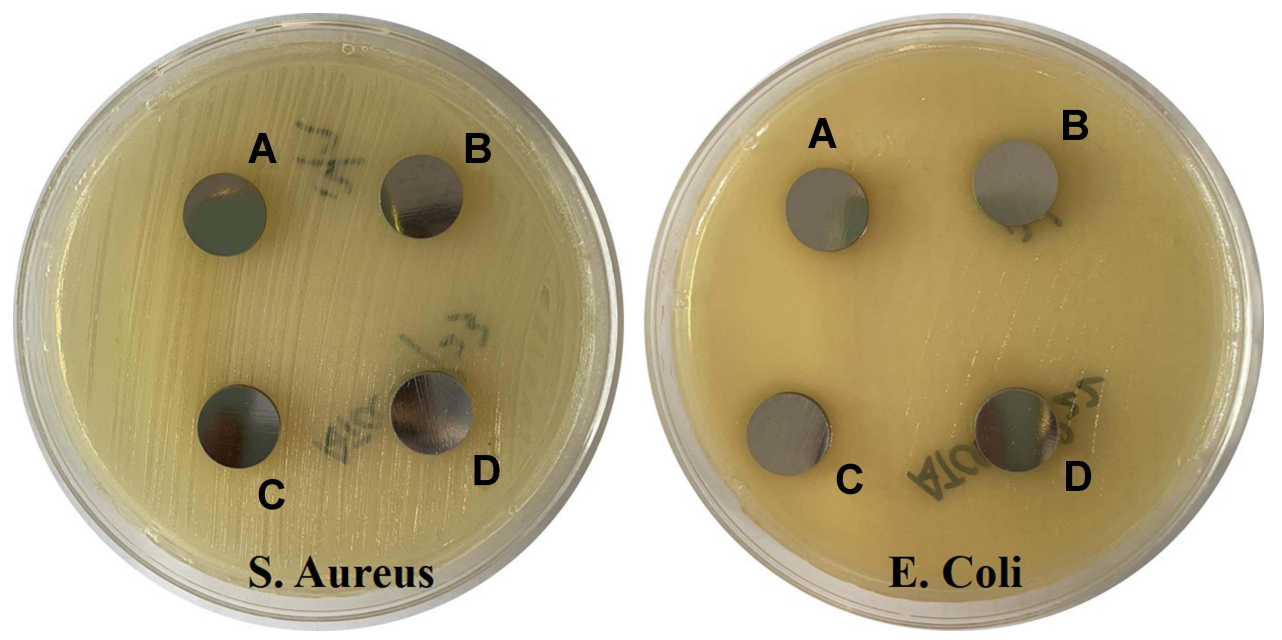

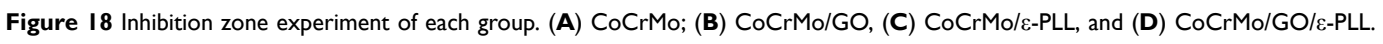
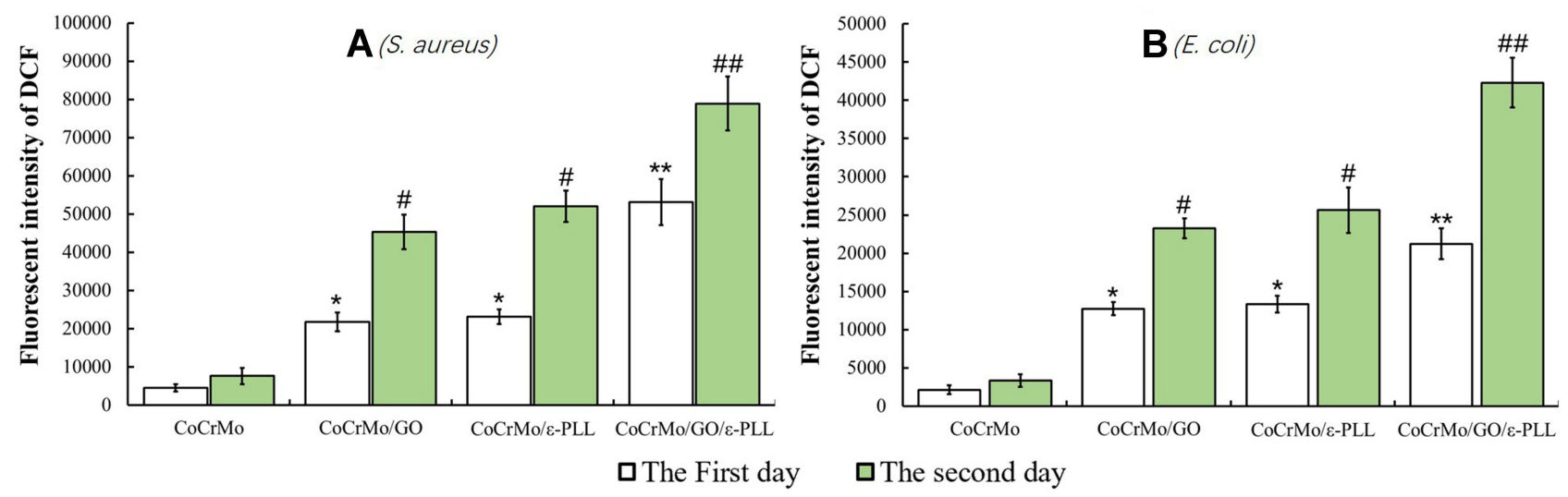

Figure 19 Fluorescence intensity of DCF on each group (*and \# $\mathrm{P}<0.05$, ** and \#\# $\mathrm{P}<0.0 \mathrm{I}$ ). (A) S. aureus. (B) E. coli.

quantify the biofilms of each group. The results of biofilm detection showed that the GO and $\varepsilon$-PLL coatings significantly inhibit the biofilm-forming ability of these two bacteria.

Cytolactase can deacetylate 2',7'-2,7-dichlorodihydrofluorescein diacetate (DCFH-DA) into nonfluorescein DCFH, which can be oxidized with intracellular ROS to fluorescein $2^{\prime}, 7^{\prime}$-dichlorofluorescein (DCF). Therefore, the fluorescence intensity of DCF can reflect the level of ROS to some extent. ${ }^{46}$ In this study, ROS levels were significantly increased in the experimental group, suggesting that ROS levels are one of the main antibacterial mechanisms of GO and $\varepsilon$-PLL.

Observation of appearance morphology showed that in the GO group, cell perforation was more common while in the $\varepsilon$-PLL group, cell atrophy was more common. We have previously performed a detailed review of the antibacterial mechanisms of $\mathrm{GO}^{37}$ on the surface of the material. In addition to oxidative stress, GO reduces microbial activity through physical damage while $\varepsilon$-PLL mainly destroys bacteria through electrostatic action and affects cell membrane permeability leading to cell disintegration (Figure 20). ${ }^{41,47,48}$ Whether the hydrophilicity and hydrophobicity of the surface of the material are one of the mechanisms affecting the antibacterial activity of the surface has not yet been determined. The results of this study show that antibacterial activity on the surface of the material has nothing to do with the hydrophilicity and hydrophobicity of the surface.

Coating materials can generally be divided into two categories: those that combine antibiotics with polymers and those that rely on the intrinsic properties of the material to kill or repel bacteria on the contact surface. ${ }^{15}$ The antimicrobial properties of the surface of the material can be generally divided into four categories: anti-adhesive surfaces, contact-killing/inhibitory surfaces, releasing- 


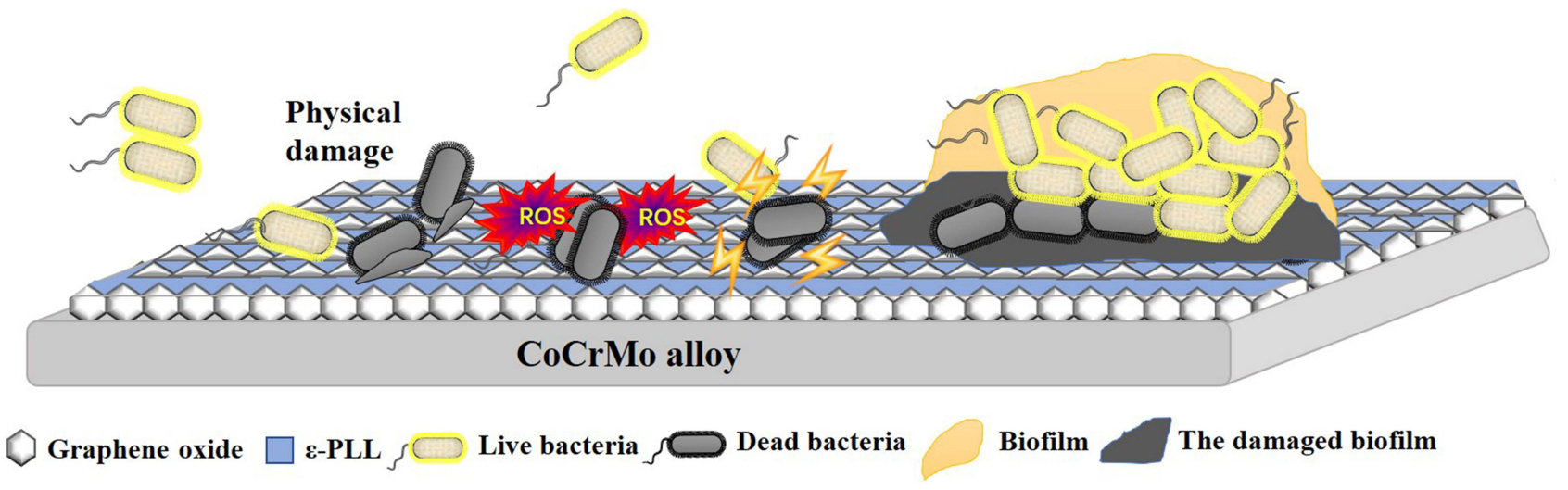

Figure 20 Antimicrobial and biofilm resistance mechanisms of GO and $\varepsilon$-PLL on CoCrMo alloy.

killing/inhibitory surfaces, and remote controllable bactericidal surfaces. In this study, we found that after coating the adhesion rate of bacteria on the surface of the material was reduced and a large number of bacteria that had adhered to it died. In the bacteriostasis zone test, we did not find the existence of inhibition zone. So, the experimental group did not kill the bacteria by releasing something. Therefore, in terms of classification, the coatings in this study belong to intrinsic properties of the material to kill or repel bacteria, its anti-adhesive and contact-killing /inhibitory surfaces.

However, regardless of the kind of coating, its adhesion strength is a problem that cannot be ignored. The elastic modulus of each group was measured by nanoindentation. In the experimental group containing GO, both the elastic modulus and Vickers hardness decreased, possibly because GO is not a single-layer structure and the combination between layers is not very strong. However, the application of $\varepsilon$-PLL solved this problem well. Compared with the control group, the elastic modulus and Vickers hardness of the group containing $\varepsilon-P L L$ were not significantly different. Here, $\varepsilon$-PLL may act as an adhesive, securing itself and GO to the surface of the alloy. However, not all antibacterial coatings need to adhere firmly to the substrate surface.

Taking the example of an artificial joint prosthesis, it is well-known that the interface of artificial joint prosthesis is generally divided into moving interface and fixed interface. Usually, infection mainly occurs at the fixed interface, while wear mainly occurs at the moving interface. In the case of artificial knee prostheses, the fixation interface, especially the interface between the polyethylene pad and the tibial plateau, is one of the most common sites for bacterial breeding. Therefore, exterminating the infection of fixed interface is equivalent to solving the infection of artificial joint prostheses to a large extent. In this study, we successfully prepared the coating and demonstrated that the coating has antibacterial and antibiofilm effects. However, for further optimization of the coating process, there is still a long way to go before it is applied in clinical practice.

\section{Conclusion}

By exploring and optimizing experimental parameters, GO and $\varepsilon$-PLL coatings were successfully prepared on a $\mathrm{CoCrMo}$ alloy by electrodeposition. The results show that the coating not only has good mechanical properties, but also has obvious antibacterial and antibiofilm effects to S. aureus and E. coli in vitro. At a time when prosthesis-related infection is becoming increasingly serious, the coating material provides a promising new approach to the problem of implant infection in orthopedics and stomatology.

\section{Acknowledgments}

This work was supported by the National Natural Science Foundation of China (82072402 and 81672189) and the Natural Science Foundation of Shaanxi Province (2020JQ-965).

\section{Disclosure}

The authors report no conflicts of interest in this work.

\section{References}

1. Chouchani C, Rolain JM, Ghrairi T. Recent advances in antimicrobial and bacterial resistance. Int J Antimicrob Agents. 2018;52(6):869-870. doi:10.1016/j.ijantimicag.2018.11.021 
2. Zhang G, Zhang X, Yang Y, et al. Dual light-induced in situ antibacterial activities of biocompatibleTiO2/MoS2/PDA/RGD nanorod arrays on titanium. Biomater Sci. 2020;8(1):391-404. doi:10.1039/ C9BM01507H

3. Rahim MI, Szafrański SP, Ingendoh-Tsakmakidis A, Stiesch M, Mueller PP. Evidence for inoculum size and gas interfaces as critical factors in bacterial biofilm formation on magnesium implants in an animal model. Colloids Surf B Biointerfaces. 2020;186:110684. doi:10.1016/j.colsurfb.2019.110684

4. Lee JK, Park SC, Hahm KS, Park Y. A helix-PXXP-helix peptide with antibacterial activity without cytotoxicity against MDRPA-infected mice. Biomaterials. 2014;35(3):1025-1039. doi:10.1016/j.biomaterials. 2013.10.035

5. Wu S, Liu Y, Zhang H, Lei L. A new transformation method with nanographene oxides of antisense carrying yycG RNA improved antibacterial properties on methicillin-resistant Staphylococcus aureus biofilm. J Vet Med Sci. 2019;81(10):1540-1546. doi:10.1292/ jvms.

19-0216

6. Kumar P, Huo P, Zhang R, Liu B. Antibacterial properties of graphene-based nanomaterials. Nanomaterials. 2019;9:5. doi:10.33 90/nano9050737

7. Rong L, Shen X, Wang B, Mao Z, Feng X, Sui X. Antibacterial thyme oil-loaded organo-hydrogels utilizing cellulose acetoacetate as reactive polymer emulsifier. Int J Biol Macromol. 2020;147:18-23. doi: 10 . 1016/j.ijbiomac.2020.01.052

8. Zeng M, Xu J, Luo Q, et al. Constructing antibacterial polymer nanocapsules based on pyridine quaternary ammonium salt Mater Sci Eng C. 2020;108:110383. doi:10.1016/j.msec.2019.11 0383

9. Weiguo X, Shujun D, Yuping H, Shuqiang L, Yang L. Hydrogels as Antibacterial Biomaterials. Curr Pharm Des. 2018;2:548.

10. Hamedi S, Shojaosadati SA, Najafi V, Alizadeh V. A novel double-network antibacterial hydrogel based on aminated bacterial cellulose and schizophyllan. Carbohydr Polym. 2020;1:229.

11. Lu X, Liu J, Gou L, et al. Designing melittin-graphene hybrid complexes for enhanced antibacterial activity. Adv Healthc Mater. 2019;8 (9):e1801521. doi:10.1002/adhm.201801521

12. Martínez M, Polizzotto A, Flores N, Semorile L, Maffía PC. Antibacterial, anti-biofilm and in vivo activities of the antimicrobial peptides P5 and P6.2. Microb Pathog. 2020;139:103886. doi:10. 1016/j.micpath.2019.103886

13. Chernousova S, Epple M. Silver as antibacterial agent: ion, nanoparticle, and metal. Angew Chem Int Ed Engl. 2013;52(6):1636-1653. doi:10.1002/anie.201205923

14. Xu T, Chen Z, Chen Z, et al. Development of the Biomaterials technology for the infection resistance. Curr Pharm Des. 2018;24 (8):886-895. doi:10.2174/1381612824666180307121716

15. Cyphert EL, von Recum HA. Emerging technologies for long-term antimicrobial device coatings: advantages and limitations. Exp Biol Med (Maywood). 2017;242(8):788-798. doi:10.1177/153537021668 8572

16. Plecko M, Sievert C, Andermatt D, et al. Osseointegration and biocompatibility of different metal implants-a comparative experimental investigation in sheep. BMC Musculoskelet Disord. 2012;13:32. doi:10.1186/1471-2474-13-32

17. Toh WQ, Tan X, Bhowmik A, Liu E, Tor SB. Tribochemical characterization and tribocorrosive behavior of cocrmo alloys: a review. Materials. 2017;11(1). doi:10.3390/ma11010030

18. Szarek A, Stradomski G, Łukomska-szarek J, Rydz D, Wolański W, Joszko K. Wear Morphology on the Surfaces of CoCrMo Unicompartmental Knee Joint Endoprostheses as Elements of Metal-Metal Friction Nodes. Materials. 2020;13:12. doi:10.3390/ ma13122689
19. Shukla K, Sugumaran AA, Khan I, Ehiasarian AP, Hovsepian PE. Low pressure plasma nitrided CoCrMo alloy utilising HIPIMS discharge for biomedical applications. J Mech Behav Biomed Mater. 2020;111:104004. doi:10.1016/j.jmbbm.2020.104004

20. Stojanović B, Bauer C, Stotter C, et al. Tribocorrosion of a CoCrMo alloy sliding against articular cartilage and the impact of metal ion release on chondrocytes. Acta Biomater. 2019;94:597-609. doi:10.1016/j.actbio.2019.06.015

21. Yao L, Min G, Han L, et al. Anti-infective application of graphene-like silicon nanosheets via membrane destruction. $A d v$ Healthc Mater. 2020;9:e1901375.

22. Assaad K, Ayoub George M, Lilian M. Antibacterial activity of chitosan nano-composites and carbon nanotubes: a review. Sci Total Environ. 2019;668:566.

23. Lin S, Jiongrun C, Lijing T, et al. The antibacterial applications of graphene and its derivatives. Small. 2016;12(31):4165-4184. doi:10. 1002/smll.201601841

24. Pandit S, Gaska K, Mokkapati V. et al. Precontrolled alignment of graphite nanoplatelets in polymeric composites prevents bacterial attachment. Small;2020. e1904756. doi:10.1002/smll.201904756

25. Sun X, Liu Z, Welsher K, et al. Nano-graphene oxide for cellular imaging and drug delivery. Nano Res. 2008;1(3):203-212. doi:10. 1007/s12274-008-8021-8

26. $\mathrm{Hu} \mathrm{W}$, Peng $\mathrm{C}$, Luo $\mathrm{W}$, et al. Graphene-based antibacterial paper. ACS Nano. 2010;4(7):4317-4323. doi:10.1021/nn101097v

27. Yoshida T, Nagasawa T. epsilon-Poly-L-lysine: microbial production, biodegradation and application potential. Appl Microbiol Biotechnol. 2003;62(1):21-26. doi:10.1007/s00253-003-1312-9

28. Shi C, He Y, Feng X, Fu D. $\varepsilon$-Polylysine and next-generation dendrigraft poly-L-lysine: chemistry, activity, and applications in biopharmaceuticals. J Biomater Sci Polym Ed. 2015;26(18):1343-1356. doi:10.1080/09205063.2015.1095023

29. Fürsatz M, Skog M, Sivlér P, et al. Functionalization of bacterial cellulose wound dressings with the antimicrobial peptide $\varepsilon$-poly -L-Lysine. Biomed Mater. 2018;13(2):025014. doi:10.1088/1748$605 X / a a 9486$

30. Jin J, Zhang L, Shi M, Zhang Y, Wang Q. Ti-GO-Ag nanocomposite: the effect of content level on the antimicrobial activity and cytotoxicity. Int J Nanomedicine. 2017;12:4209-4224. doi:10.2147/ IJN.S134843

31. Qiu J, Liu L, Zhu H, Liu X. Combination types between graphene oxide and substrate affect the antibacterial activity. Bioact Mater. 2018;3(3):341-346. doi:10.1016/j.bioactmat.2018.05.001

32. Kania R, Vironneau P, Dang H, et al. Bacterial biofilm in adenoids of children with chronic otitis media. Part I: a case control study of prevalence of biofilms in adenoids, risk factors and middle ear biofilms. Acta Otolaryngol. 2019;139(4):345-350. doi:10.1080/ 00016489.2019.1571282

33. Ghilini F, Rodríguez González MC, Miñán AG, et al. Highly stabilized nanoparticles on poly-l-lysine-coated oxidized metals: a versatile platform with enhanced antimicrobial activity. ACS Appl Mater Interfaces. 2018;10(28):23657-23666. doi:10.1021/acsami.8b 07529

34. Varoni E, Canciani E, Palazzo B, et al. Effect of Poly-L-Lysine coating on titanium osseointegration: from characterization to in vivo studies. J Oral Implantol. 2015;41(6):626-631. doi:10.1563/ AAID-JOI-D-13-00036

35. Christensen GD, Simpson WA, Younger JJ, et al. Adherence of coagulase-negative staphylococci to plastic tissue culture plates: a quantitative model for the adherence of staphylococci to medical devices. J Clin Microbiol. 1985;22(6):996-1006. doi:10.1128/ jcm.22.6.996-1006.1985

36. Josse J, Valour F, Maali Y, et al. Interaction Between staphylococcal biofilm and bone: how does the presence of biofilm promote prosthesis loosening. Front Microbiol. 2019;10:1602. doi:10.3389/ fmicb.2019.01602 
37. Guihua Cao JY, Xiaoxuan Ning QZ, Qi WLB, Yumin Zhang YH, Guo J. Antibacterial and antibiofilm properties of graphene and its derivatives. Colloids Surf B Biointerfaces. 2021;22: 111588 .

38. Dubey N, Ellepola K, Decroix F, et al. Graphene onto medical grade titanium: an atom-thick multimodal coating that promotes osteoblast maturation and inhibits biofilm formation from distinct species. Nanotoxicology. 2018;12(4):274-289. doi:10.1080/17435390.2018. 1434911

39. Glavis-Bloom J, Vasher S, Marmor M, et al. Candida and cardiovascular implantable electronic devices: a case of lead and native aortic valve endocarditis and literature review. Mycoses. 2015;58(11): 637-641. doi:10.1111/myc. 12391

40. Pilz M, Staats K, Tobudic S, et al. Zirconium nitride coating reduced staphylococcus epidermidis biofilm formation on orthopaedic implant surfaces: an in vitro study. Clin Orthop Relat Res. 2019;477(2): 461-466. doi:10.1097/CORR.0000000000000568

41. Lin L, Gu Y, Li C, Vittayapadung S, Cui H. Antibacterial mechanism of \&Poly-lysine against Listeria monocytogenes and its application on cheese. Food Control. 2018;91(1):76-84. doi:10.1016/j.foodcont.2018.03.025

42. Jakobsen SS, Larsen A, Stoltenberg M, Bruun JM, Soballe K. Effects of as-cast and wrought Cobalt-Chrome-Molybdenum and Titanium-Aluminium-Vanadium alloys on cytokine gene expression and protein secretion in J774A.1 macrophages. Eur Cell Mater. 2007;14:45-54. doi:10.22203/eCM.v014a05
43. Kubacki GW, Sivan S, Gilbert JL. Electrosurgery Induced Damage to Ti-6Al-4V and CoCrMo Alloy Surfaces in Orthopedic Implants In Vivo and In Vitro. J Arthroplasty. 2017;32(11):3533-3538. doi:10. 1016/j.arth.2017.06.015

44. Bradley D, Xiaoning L, Landis Ryan F, et al. Nanoparticle-Stabilized Capsules for the Treatment of Bacterial Biofilms. ACS Nano. 2015;9:8.

45. Yong L, Busscher Henk J, Bingran Z, et al. Surface-adaptive, antimicrobially loaded, micellar nanocarriers with enhanced penetration and killing efficiency in staphylococcal biofilms. ACS Nano. 2016;10:4.

46. Qiu J, Liu L, Chen B, et al. Graphene oxide as a dual $\mathrm{Zn} / \mathrm{Mg}$ ion carrier and release platform: enhanced osteogenic activity and antibacterial properties. J Mater Chem B. 2018;6(13):2004-2012. doi:10. 1039/C8TB00162F

47. Liu K, Zhou X, Fu M. Inhibiting effects of epsilon-poly-lysine (ع-PL) on Pencillium digitatum and its involved mechanism. Postharvest Biol Technol. 2017;123:94-101. doi:10.1016/j.postharvbio.2016.08.015

48. Chang Y, McLandsborough L, McClements DJ. Physicochemical properties and antimicrobial efficacy of electrostatic complexes based on cationic e-polylysine and anionic pectin. J Agric Food Chem. 2011;59(12):6776-6782. doi:10.1021/jf201162g
International Journal of Nanomedicine

\section{Publish your work in this journal}

The International Journal of Nanomedicine is an international, peerreviewed journal focusing on the application of nanotechnology in diagnostics, therapeutics, and drug delivery systems throughout the biomedical field. This journal is indexed on PubMed Central, MedLine, CAS, SciSearch ${ }^{\circledR}$, Current Contents ${ }^{\mathbb{R}} /$ Clinical Medicine,

\section{Dovepress}

Journal Citation Reports/Science Edition, EMBase, Scopus and the Elsevier Bibliographic databases. The manuscript management system is completely online and includes a very quick and fair peer-review system, which is all easy to use. Visit http://www.dovepress.com/ testimonials.php to read real quotes from published authors. 